\title{
Kantorovich problems and conditional measures depending on a parameter
}

\section{Vladimir I. Bogachev ${ }^{a, b}$, $]$, Ilya I. Malofeev ${ }^{a}$}

\author{
${ }^{a}$ Department of Mechanics and Mathematics, Moscow State University, 119991 \\ Moscow, Russia \\ ${ }^{b}$ National Research University Higher School of Economics, Moscow, Russia
}

\begin{abstract}
We study measurable dependence of measures on a parameter in the following two classical problems: constructing conditional measures and the Kantorovich optimal transportation. For parametric families of measures and mappings we prove the existence of conditional measures measurably depending on the parameter. A particular emphasis is made on the Borel measurability (which cannot be always achieved). Our second main result gives sufficient conditions for the Borel measurability of optimal transports and transportation costs with respect to a parameter in the case where marginal measures and cost functions depend on a parameter. As a corollary we obtain the Borel measurability with respect to the parameter for disintegrations of optimal plans. Finally, we show that the Skorohod parametrization of measures by mappings can be also made measurable with respect to a parameter.
\end{abstract}

Keywords: Kantorovich problem, conditional measure, weak convergence, measurable dependence on a parameter, Skorohod representation

AMS MSC 2010: 28C15, 60G57, 46G12

\section{INTRODUCTION}

We recall that, given two probability spaces $\left(X, \mathcal{B}_{X}, \mu\right)$ and $\left(Y, \mathcal{B}_{Y}, \nu\right)$ and a nonnegative $\mathcal{B}_{X} \otimes \mathcal{B}_{Y}$-measurable function $h$ on $X \times Y$ (called a cost function), the associated Kantorovich problem is to find the infimum of the integral

$$
I_{h}(\sigma):=\int h d \sigma
$$

over all probability measures $\sigma$ on $\mathcal{B}_{X} \otimes \mathcal{B}_{Y}$ with projections $\mu$ and $\nu$ on the factors. This infimum is denoted by

$$
K_{h}(\mu, \nu)
$$

and called the transportation cost for $h, \mu, \nu$. If this infimum is attained (is a minimum, which happens under broad assumptions), then the minimizing measures are called optimal measures (and also optimal plans or optimal transports). The measures $\mu$ and $\nu$ are called marginal distributions. There is an extensive literature on this subject, see, e.g., 2], [14, [24], [35], 41], and [42]. This paper was motivated by several questions posed by Sergey Kuksin about measurable dependence of Kantorovich optimal transportation plans on a parameter in optimal transportation problems depending on a parameter.

Suppose now that $(T, \mathcal{T})$ is a measurable space and for each $t$ we have marginal probability measures $\mu_{t}$ and $\nu_{t}$ (which depend on $t$ measurably in the sense that the

${ }^{1}$ Corresponding author, vibogach@mail.ru. 
functions $t \mapsto \mu_{t}(A)$ are $\mathcal{T}$-measurable for all $A \in \mathcal{B}_{X}$ and similarly for $\left.\nu_{t}\right)$ and that also the cost function depends on the parameter $t$, i.e.,

$$
h: T \times X \times Y \rightarrow[0,+\infty)
$$

is a $\mathcal{T} \otimes \mathcal{B}_{X} \otimes \mathcal{B}_{Y}$-measurable function. We set

$$
h_{t}(x, y):=h(t, x, y) .
$$

Thus, we obtain a Kantorovich problem with a parameter. Dependence on a parameter appears even for a single cost function if only marginal distributions depend on $t$. The question is whether the infimum depends measurably on $t$ and there are optimal plans $\sigma_{t}$ measurably depending on $t$.

Several results have already been obtained in this situation. Villani [42] considered the situation where only the marginal distributions depend on a parameter (and are Borel measures on Polish spaces), but the cost function does not. Dedecker, Prieur and Raynaud De Fitte [18] studied the case of metric-type cost functions (such that $h(x, y)=\sup |u(x)-u(y)|$, where sup is taken over bounded continuous functions $u$ with $|u(x)-u(y)| \leq h(x, y)$ ) on rather general spaces (including completely regular Souslin spaces) and established the existence of a measurable selection of an optimal measure and the measurability of the Kantorovich minimum, however, this measurability is with respect to the $\sigma$-algebra of universally measurable sets, not with respect to the Borel $\sigma$-algebra. Similar results are also contained in [16, Sections 3.4 and 7.1]. Zhang [43] gave a result for continuous cost functions on Polish spaces $X$ and $Y$ and an arbitrary measurable space $T$, but the justification contains a gap and the really proved fact is this: if we consider the space $M=C(X \times Y)$ with the Borel $\sigma$-algebra corresponding to the metric

$$
d_{M}(f, g)=\sum_{n=1}^{\infty} 2^{-n} \min \left(1, \sup _{z \in B_{n}}|f(z)-g(z)|\right),
$$

where $\left\{B_{n}\right\}$ is a fixed sequence of increasing balls with the union $X \times Y$, and to every triple $(h, \mu, \nu)$ with a nonnegative function $h \in M$ we associate the set $\operatorname{Opt}(h, \mu, \nu)$ of all optimal measures, then there is a selection of an optimal measure measurable with respect to the $\sigma$-algebras $\mathcal{B}(M) \otimes \mathcal{B}(\mathcal{P}(X)) \otimes \mathcal{B}(\mathcal{P}(Y))$ and $\mathcal{B}(\mathcal{P}(X \times Y))$. However, this does not imply the measurability claimed in [43] (the measurability with respect to $\mathcal{T}$ for a general $\sigma$-algebra $\mathcal{T}$ ), because the mapping $t \mapsto h(t, \cdot, \cdot)$ can fail to be measurable with respect to $\mathcal{T}$ and $\mathcal{B}(M)$ under the only assumption that $h$ is measurable on $T \times X \times Y$. The point is that for a noncompact space $Z$ the Borel $\sigma$-algebra of the space $C_{b}(Z)$ with its sup-norm is not generated by evaluation functionals $z \mapsto f(z)$ (see Remark 5.12 below). A consequence of this in the situation of [43] is that the assumed measurability of the cost function is not sufficient for the applicability of the established selection result. However, it will be shown below in Theorem 4.2 that the main result of [43] is valid. Moreover, we show that optimal transports can be made Borel measurable with respect to the parameter for lower semicontinuous cost functions in place of continuous ones, provided that $T$ is a Souslin space with its Borel $\sigma$-algebra.

In the study of optimal plans one often deals with conditional measures. It is, of course, a question of independent interest to study conditional measures depending on a parameter (and this question was also suggested to us by Sergey Kuksin). The general framework for conditional measures is this: given a measure $\mu$ on a space $X$ and a measurable mapping $f$ of $X$ onto another measurable space $Y$, we are looking 
for measures $\mu^{y}$ concentrated on the level sets $f^{-1}(y)$ for $y \in Y$ such that $\mu$ has the form

$$
\mu=\int_{Y} \mu^{y} \nu(d y)
$$

where $\nu=\mu \circ f^{-1}(d y)$ is the image of $\mu$ under $f$ (or some other natural measure on $Y$ ). Below we recall a precise definition. Again, once $\mu$ and $f$ depend on a parameter $t$, the question is whether one can pick conditional measures $\mu_{t}^{y}$ measurably depending on $t$. A positive result was obtained in [33] (where a sketch of the proof was given), but, as above, this result is in terms of measurability with respect to the extensions of Borel $\sigma$-algebras generated by Souslin sets. Below (see Theorem 3.4) we provide all technical details for a more general result and complement this result by sufficient conditions for the Borel measurability (Theorem 3.5). Moreover, the existence of jointly (i.e., in both variables) Borel measurable conditional measures depending on a parameter is shown (see Proposition 3.10) to be equivalent to the existence of jointly Borel measurable right inverse mappings, similarly to the result of Blackwell and Ryll-Nardzewski [8] in the case of measures and mappings without parameters. It is worth noting that although sets from the $\sigma$-algebra generated by Souslin sets remain measurable with respect to all Borel measures, their weak point is that continuous images (say, projections) of such sets can fail to be measurable. This is one of motivations to desire the Borel measurability.

Both problems (dependence on a parameter for optimal plans and conditional measures) have some common features and are strongly connected with measurable choice theorems. It will be more convenient to start with conditional measures, which is done in Section 3. In Section 4 we discuss optimal plans and formulate our main results, which are proved in Section 5 along with a number of auxiliary results.

Finally, in Section 6 we consider along the same lines the classical result going back to Skorohod and giving a parametrization of Borel probability measures $\mu$ on a Polish space $X$ by Borel mappings $\xi_{\mu}:[0,1] \rightarrow X$ such that $\mu$ is the image of Lebesgue measure $\lambda$ under $\xi_{\mu}$ and measures $\mu_{n}$ converge weakly to $\mu$ if and only if the mappings $\xi_{\mu_{n}}$ converge to $\xi_{\mu}$ almost surely. We show that there is a version of $\xi_{\mu}$ such that the function $(\mu, t) \mapsto \xi_{\mu}(t)$ is Borel measurable on $[0,1] \times \mathcal{P}(X)$. It follows that for any family of measures $\mu_{\omega}$ measurably depending on a parameter $\omega$, the function $(\omega, t)=\xi_{\mu_{\omega}}(t)$ is jointly Borel measurable.

\section{Notation AND TERMinOlOGY}

We shall consider Borel measures on complete separable metric spaces and in some results on Souslin spaces. So we briefly recall these concepts and some related objects.

Let $X$ be a topological space. Its Borel $\sigma$-algebra, denoted by $\mathcal{B}(X)$, is the smallest $\sigma$-algebra containing all open sets. A real function $f$ on $X$ is called Borel measurable if the sets $\{x: f(x)<c\}$ are Borel for all $c$. A mapping $f$ from $X$ to a topological space $Y$ is called Borel measurable if $f^{-1}(B)$ is a Borel set for every Borel set $B \subset Y$. For $Y=\mathbb{R}$ this is equivalent to the aforementioned definition.

The space of bounded continuous functions on $X$ with its sup-norm is denoted by $C_{b}(X)$. The space of bounded Borel measurable functions with the same norm is denoted by $B_{b}(X)$. 
If $(T, \mathcal{T})$ is a measurable space (i.e., $\mathcal{T}$ is a $\sigma$-algebra), then a mapping $f: T \rightarrow X$ is called $\mathcal{T}$-measurable (or $(\mathcal{T}, \mathcal{B}(X))$-measurable) if $f^{-1}(B) \in \mathcal{T}$ for all $B \in \mathcal{B}(X)$. The Borel measurability is a particular case of this definition.

A space homeomorphic to a complete separable metric space is called Polish. A Hausdorff space that is the image of a complete separable metric space under a continuous mapping is called Souslin or analytic (see, e.g., [9], 29]). If such a mapping can be found one-to-one, then $X$ is called a Luzin space. A Hausdorff space $X$ is completely regular if for every point $x \in X$ and every open set $U$ containing $x$ there is a continuous function $f: X \rightarrow[0,1]$ such that $f(x)=1$ and $f=0$ outside $U$.

Borel sets in Polish spaces are Souslin (and even Luzin) spaces; Borel sets in Souslin spaces are also Souslin. However, unlike the case of Borel sets, the complement of a Souslin set $A$ in a Polish space is not always Borel, moreover, it can be Borel only if $A$ itself is Borel. For this reason, the $\sigma$-algebra $\sigma(\mathcal{S}(X))$ generated by the class $\mathcal{S}(X)$ of all Souslin sets in $X$ is much larger than the Borel $\sigma$-algebra (although its cardinality is the continuum for infinite spaces); for example, in typical cases it is not countably generated (see [9, Example 6.5.9]).

Souslin sets belong to the Lebesgue completion of the Borel $\sigma$-algebra for every Borel measure on a Souslin space (i.e., they are universally measurable), hence the same is true for the generated $\sigma$-algebra $\sigma(\mathcal{S}(X))$. However, this $\sigma$-algebra is not stable under the Souslin operation, unlike the completion of the Borel $\sigma$ algebra (see [9, p. 66]) and unlike the $\sigma$-algebra of universally measurable sets. The images and preimages of Souslin sets under Borel mappings are Souslin. For Borel sets, only preimages are Borel: it was shown by Souslin that the projection of a Borel set in $\mathbb{R}^{2}$ can fail to be Borel. Next, the preimages of sets in $\sigma(\mathcal{S}(X))$ under Borel mappings are also in $\sigma(\mathcal{S}(X))$. This is not true for their images even under continuous mappings: the projection of the complement of a Souslin set need not belong to $\sigma(\mathcal{S}(X)$ ) (for example, the projection of the complement of a Souslin set need not be Lebesgue measurable).

Borel measures are finite (possibly, signed) measures on $\mathcal{B}(X)$. A signed Borel measure $\mu$ can be written as $\mu=\mu^{+}-\mu^{-}$, where $\mu^{+}$and $\mu^{-}$are mutually singular nonnegative Borel measures. The measure $|\mu|=\mu^{+}+\mu^{-}$is called the total variation of $\mu$ and the number $\|\mu\|=|\mu|(X)$ is called the total variation norm or the variation norm. We mostly deal with probability measures.

A Borel measure $\mu$ is called Radon if for every Borel set $B$ and every $\varepsilon>0$ there is a compact set $K_{\varepsilon} \subset B$ such that $|\mu|\left(B \backslash K_{\varepsilon}\right)<\varepsilon$. On a Souslin space all Borel measures are Radon.

The image of a measure $\mu$ on $X$ under a measurable mapping $f: X \rightarrow Y$ is denoted by $\mu \circ f^{-1}$ and defined by the equality

$$
\left(\mu \circ f^{-1}\right)(E)=\mu\left(f^{-1}(E)\right), E \in \mathcal{B}(Y) .
$$

Let $\mathcal{P}(X)$ be the space of all Borel probability measures on a completely regular space $X$. Recall that the weak topology on the whole space $\mathcal{M}(X)$ of signed Borel measures is generated by duality with $C_{b}(X)$, i.e., is defined by means of seminorms

$$
\mu \mapsto\left|\int_{X} f d \mu\right|,
$$

where $f \in C_{b}(X)$. Throughout the spaces of measures will be considered with the weak topology and the corresponding Borel structure. 
If $X$ is a completely regular Souslin space, then $\mathcal{M}(X)$ and $\mathcal{P}(X)$ are also completely regular Souslin spaces; if $X$ is a Polish space, then $\mathcal{P}(X)$ is also Polish (but $\mathcal{M}(X)$ is not in nontrivial cases) and if $X$ is a Luzin space, then so is $\mathcal{P}(X)$. These facts can be found in [9, Chapter 8] or in [12, Chapter 5].

We shall employ Prohorov's condition for compactness in $\mathcal{M}(X)$ : a set $M$ has compact closure in $\mathcal{M}(X)$ if it is bounded in variation and uniformly tight, which means that for every $\varepsilon>0$ there is a compact set $K \subset X$ such that $|\mu|(X \backslash K) \leq \varepsilon$ for all $\mu \in M$. If $X$ is a Polish space, then this condition is also necessary.

For a completely regular Souslin space $X$, a mapping $m:(\Omega, \mathcal{E}) \rightarrow \mathcal{P}(X)$ from a measurable space $(\Omega, \mathcal{E})$ is measurable if and only if all functions

$$
\omega \mapsto \int_{X} \varphi(x) m(\omega)(d x), \quad \varphi \in C_{b}(X)
$$

are $\mathcal{E}$-measurable. This is also equivalent to the $\mathcal{E}$-measurability of all functions

$$
\omega \mapsto \int_{X} \varphi_{n}(x) m(\omega)(d x)
$$

for any countable family of functions $\varphi_{n} \in C_{b}(X)$ of the form $\varphi_{n}=p\left(f_{1}, \ldots, f_{k}\right)$, where $p$ is a polynomial on $\mathbb{R}^{k}$ with rational coefficients and $\left\{f_{j}\right\} \subset C_{b}(X)$ is a sequence separating the points in $X$ (such sequences exist for all completely regular Souslin spaces, see [9, Theorem 6.7.7]). Recall that any sequence of Borel functions separating points of a Souslin space generates the Borel $\sigma$-algebra of this space (see [9, Theorem 6.8.9]). It is readily verified by the monotone class theorem (see [9, Theorem 6.7.7] and [33]) that this measurability is equivalent to the $\mathcal{E}$-measurability of all functions

$$
\omega \mapsto m(\omega)(B), B \in \mathcal{B}(X)
$$

Recall that a mapping $\Psi$ from a measurable space $(T, \mathcal{T})$ to the set of nonempty subsets of a topogical space $X$ is called measurable if for every open set $U \subset X$ the set $\{t: \Psi(t) \cap U \neq \emptyset\}$ belongs to $\mathcal{T}$.

The space $\mathcal{K}(X)$ of nonempty compact subsets of a complete metric space $X$ is equipped with the Hausdorff distance

$$
d_{H}(A, B)=\inf \{r>0: \operatorname{dist}(a, B)<r, \operatorname{dist}(b, A)<r \forall a \in A, b \in B\} .
$$

It is known that this space is complete and separable (and is compact if $X$ is compact), see [17].

\section{Conditional measures Depending on a Parameter}

We first address the problem of conditional measures. A general discussion can be found in [9, Chapter 10]; see also [1, [10], 25], 27], 28], [37, and [39. Connections between conditional measures and surface measures are considered in [15] and [11].

It is known that, whenever $\mu$ is a Borel probability measure on a Souslin space $X$ and $f$ is a Borel mapping from $X$ to a Souslin space $Y$, the level sets $f^{-1}(y)$ can be equipped with Borel probability measures $\mu^{y}$, called conditional measures generated by $f$, possessing the following three properties:

1) the measure $\mu^{y}$ is concentrated on the set $f^{-1}(y)$ for each $y \in f(X)$, i.e.,

$$
\mu^{y}\left(f^{-1}(y)\right)=1, y \in f(X)
$$

2) the functions

$$
y \mapsto \mu^{y}(B), \quad B \in \mathcal{B}(X),
$$


are measurable with respect to the $\sigma$-algebra $\sigma(\mathcal{S}(X))$ generated by the class of Souslin sets in $X$,

3) if $B \subset X$ and $E \subset Y$ are Borel sets, then

$$
\mu\left(B \cap f^{-1}(E)\right)=\int_{E} \mu^{y}(B) \mu \circ f^{-1}(d y) .
$$

Conditional measures with properties 1)-3) are called regular proper conditional probabilities, the term "proper" refers to condition 1 ).

It should be noted that due to condition 1 ) the last equality for all $B$ is equivalent to its special case with $E=Y$ :

$$
\mu(B)=\int_{Y} \mu^{y}(B) \mu \circ f^{-1}(d y) .
$$

Indeed, replacing $B$ by $B \cap f^{-1}(E)$ we have $\mu^{y}\left(B \cap f^{-1}(E)\right)=\mu^{y}(B)$ if $y \in E$, because $\mu^{y}$ is concentrated on $f^{-1}(y)$. If $y \notin E$, then for the same reason $\mu^{y}\left(B \cap f^{-1}(E)\right)=0$, since $f^{-1}(E) \cap f^{-1}(y)=\emptyset$. However, the equivalent formulation with $E$ is sometimes useful.

The equality in condition 3) is equivalent to the following: for every bounded Borel function $\varphi$ on $X$ and every Borel set $E \subset Y$ we have

$$
\int_{f^{-1}(E)} \varphi d \mu=\int_{E} \int_{X} \varphi(x) \mu^{y}(d x) \mu \circ f^{-1}(d y)=\int_{f^{-1}(E)} \int_{X} \varphi(x) \mu^{f(u)}(d x) \mu(d u) .
$$

As above, it suffices to have this identity for $E=Y$.

It is known (see [9]) that conditional measures are unique in the following sense: two such collections coincide for all points $y$ outside a set of measure zero with respect to the induced measure $\mu \circ f^{-1}$.

If $\mathcal{B}^{f}=\left\{f^{-1}(A): A \in \mathcal{B}(Y)\right\}$ is the $\sigma$-algebra generated by $f$, then the function

$$
E\left(\varphi \mid \mathcal{B}^{f}\right)(u)=\int_{X} \varphi(x) \mu^{f(u)}(d x)
$$

serves as the conditional expectation of $\varphi$ with respect to $\mathcal{B}^{f}$.

It is possible to modify conditions 1) and 2) as follows: the Borel measurability of all functions in 2) can be achieved at the expense of weakening condition 1) by replacing it by the condition that $\mu^{y}\left(f^{-1}(y)\right)=1$ for $\mu \circ f^{-1}$-almost all $y$. However, in the general case it is impossible to guarantee the Borel measurability of all functions $y \mapsto \mu^{y}(B)$ if the equality $\mu^{y}\left(f^{-1}(y)\right)=1$ must hold for each $y$. There are counterexamples even in the case where $X$ is a Borel set in $[0,1]$ and $f$ is a smooth function (see [8] or [9, V. 2, p. 430]). According to [8], if $X$ is a Polish space, the existence of conditional measures $\mu^{y}$ such that 1) holds and all functions $y \mapsto \mu^{y}(B)$ are Borel implies that $f(X)$ is a Borel set. A necessary and sufficient condition for the existence of such conditional measures is this: there exists a mapping $F: X \rightarrow X$ measurable with respect to $\mathcal{B}^{f}$ and $\mathcal{B}(X)$ such that $f(F(x))=f(x)$. If $f$ is surjective, this is equivalent to the existence of a Borel mapping $g: Y \rightarrow X$ that is right inverse to $f: f(g(y))=y$. Indeed, since $F$ is $\mathcal{B}^{f}$-measurable, it must be of the form $F(x)=$ $g(f(x))$ for some Borel mapping $g: Y \rightarrow X$, hence $f(g(f(x)))=f(x)$, whence $f(g(y))=y$ for all $y \in Y$. Conversely, if such $g$ exists, we can take $F(x)=g(f(x))$. Some measurability problems connected with conditional measures are discussed in [36].

Suppose now that $\mu$ and $f$ depend measurably on a parameter $z$ belonging to some Souslin space $Z$. Is it possible to pick conditional measures $\mu_{z}^{y}$ depending measurably 
on $(y, z)$ ? This question arises naturally in applications, in particular, in optimal transportation and parametric statistics (see, e.g., [34], [14], [22], and [42]). Some positive results have been recently given in [33]. Here we reinforce these results (and also give all details of proofs omitted in [33].

Throughout that $X, Y, Z$ are assumed to be completely regular Souslin spaces (in some results certain stronger assumptions are used).

Lemma 3.1. Suppose that $\psi: X \times Z \rightarrow \mathbb{R}$ is a bounded function measurable with respect to the $\sigma$-algebra $\sigma(\mathcal{S}(X)) \otimes \sigma(\mathcal{S}(Z))$. Let $z \mapsto \mu_{z}, X \rightarrow \mathcal{P}(X)$ be Borel measurable or, more generally, $(\sigma(\mathcal{S}(Z)), \mathcal{B}(\mathcal{P}(X)))$-measurable. Then the function

$$
h(z)=\int_{X} \psi(x, z) \mu_{z}(d x)
$$

is $\sigma(\mathcal{S}(Z))$-measurable on $Z$. If $\psi$ and $z \mapsto \mu_{z}$ are Borel measurable, then the function $h$ is also Borel measurable.

Proof. In the case of the $(\sigma(\mathcal{S}(Z)), \mathcal{B}(\mathcal{P}(X)))$-measurability the class $\mathcal{H}$ of all bounded $\sigma(\mathcal{S}(X)) \otimes \sigma(\mathcal{S}(Z))$-measurable functions $\psi$ for which $h$ is $\sigma(\mathcal{S}(Z))$-measurable is closed with respect to uniform limits and limits of increasing uniformly bounded sequences. Moreover, it contains all functions of the form

$$
\varphi_{1}(x) \psi_{1}(z)+\cdots+\varphi_{n}(x) \psi_{n}(z),
$$

where $\varphi_{i}$ and $\psi_{i}$ are bounded functions on $X$ and $Z$ measurable with respect to $\sigma(\mathcal{S}(X))$ and $\sigma(\mathcal{S}(Z))$, respectively. Applying the monotone class theorem, we conclude that $\mathcal{H}$ is the space of all bounded $\sigma(\mathcal{S}(X)) \otimes \sigma(\mathcal{S}(Z))$-measurable functions (see [9, Theorem 2.12.9]). In the case of Borel measurability, the same reasoning applies if we take for $\mathcal{H}$ the class of all bounded Borel measurable functions for which the corresponding function $h$ is Borel measurable.

Remark 3.2. It follows from the lemma that if we have a family of Borel sets $B_{z}$ such that the function $I_{B_{z}}(x)$ is $\sigma(\mathcal{S}(X)) \otimes \sigma(\mathcal{S}(Z))$-measurable, then the function $z \mapsto \mu_{z}\left(B_{z} \cap B\right)$ is $\sigma(\mathcal{S}(Z))$-measurable for all $B \in \sigma(\mathcal{S}(X))$ and similarly in the Borel case.

Lemma 3.3. Suppose that we have a mapping $(y, z) \mapsto \nu_{z}^{y}$ from $Y \times Z$ to $\mathcal{P}(X)$ that is measurable with respect to $\sigma(\mathcal{S}(Y \times Z))$ and $\mathcal{B}(\mathcal{P}(X))$ and a mapping

$$
(z, x) \mapsto f_{z}(x), \quad Z \times X \rightarrow Y
$$

that is measurable with respect to $\sigma(\mathcal{S}(Y \times Z))$ and $\mathcal{B}(Y)$. Then the set

$$
S=\left\{(y, z) \in Y \times Z: \nu_{z}^{y}\left(f_{z}^{-1}(y)\right)=1\right\}
$$

belongs to $\sigma(\mathcal{S}(Y \times Z))$. If both mappings are Borel measurable, then $S$ is also Borel.

Proof. The pair $(y, z)$ belongs to this set precisely when

$$
\nu_{z}^{y} \circ f_{z}^{-1}=\delta_{y} .
$$

This is equivalent to the identity

$$
\int_{X} \psi_{j}\left(f_{z}(x)\right) \nu_{z}^{y}(d x)=\psi_{j}(y)
$$

for a fixed countable family $\left\{\psi_{j}\right\} \subset C_{b}(Y)$ separating Borel measures on $Y$ (as recalled above, such collections exist for all completely regular Souslin spaces). Since $\psi_{j}$ is Borel measurable, it remains to apply Lemma 3.1 to the space $Y \times Z$. 
We now prove the existence of conditional measures measurably depending on a parameter. Our proof is a modification of the reasoning used in [33], where a somewhat stronger assumption was used, but for the reader's convenience we repeat some steps from [33] instead of referring to that paper. Another important reason for this repeating is that we also indicate some changes necessary for obtaining conditions for the Borel measurability of conditional measures, which will be the subject of the next theorem.

Theorem 3.4. Let

$$
f:(x, z) \mapsto f_{z}(x), \quad X \times Z \rightarrow Y
$$

be a Borel mapping. Suppose that for every $z \in Z$ there is a Borel probability measure $\mu_{z}$ on $X$ such that the mapping

$$
z \mapsto \mu_{z}, Z \rightarrow \mathcal{P}(X)
$$

is Borel measurable or, more generally, $(\sigma(\mathcal{S}(Z)), \mathcal{B}(\mathcal{P}(X)))$-measurable. Then, for all pairs $\left(\mu_{z}, f_{z}\right)$, there exist proper conditional probabilities $\left\{\mu_{z}^{y}\right\}_{y \in Y}$ on $X$ such that, for every Borel set $B$ in $X$, the function

$$
(y, z) \mapsto \mu_{z}^{y}(B)
$$

on $Y \times Z$ is $\sigma(\mathcal{S}(Y \times Z))$-measurable, i.e., the mapping

$$
(y, z) \mapsto \mu_{z}^{y}, \quad Y \times Z \rightarrow \mathcal{P}(X)
$$

is measurable with respect to the $\sigma$-algebra $\sigma(\mathcal{S}(Y \times Z))$.

Proof. For every point $z \in Z$, we take the measure

$$
\sigma_{z}:=\mu_{z} \circ f_{z}^{-1}
$$

on $Y$ and an increasing sequence of finite algebras $\mathcal{B}_{z, n}$ the union of which generates the $\sigma$-algebra

$$
\mathcal{B}_{z}:=f_{z}^{-1}(\mathcal{B}(Y))
$$

Without loss of generality we can assume that $\mathcal{B}_{z, n}$ is generated by some finite partition of $X$ into disjoint sets of the form

$$
A_{z, n, 1}=f_{z}^{-1}\left(B_{n, 1}\right), \ldots, A_{z, n, m_{n}}=f_{z}^{-1}\left(B_{n, m_{n}}\right),
$$

where $B_{n, 1}, \ldots, B_{n, m_{n}}$ is a finite partition of $Y$ into disjoint Borel sets such that the union of $B_{n, i}$ over all $n$ and $i$ generates $\mathcal{B}(Y)$. For the space $Y=[0,1)$ one can take $B_{n, i}=[i / n,(i+1) / n)$. In the general case there is a continuous injection $T$ of $Y$ into the countable power $[0,1]^{\infty}$ of $[0,1]$ equipped with the product topology. Since this is a compact metric space, it can be covered by finitely many balls $K_{n, i}$ of radius $1 / n$. So we take $B_{n, i}=T^{-1}\left(D_{n, i}\right)$, where $D_{n, 1}=K_{n, 1}, D_{n, i+1}=K_{n, i+1} \backslash\left(K_{n, 1} \cup \cdots \cup K_{n, i}\right)$.

The conditional measures for $\mu_{z}$ and the $\sigma$-algebra $\mathcal{B}_{z, n}$ can be written explicitly:

$$
\mu_{z, n}^{y}(A)=\sum_{i=1}^{m_{n}} \frac{\mu_{z}\left(A \cap A_{z, n, i}\right)}{\mu_{z}\left(A_{z, n, i}\right)} I_{B_{n, i}}(y),
$$

where $\mu_{z}\left(A \cap A_{z, n, i}\right) / \mu_{z}\left(A_{z, n, i}\right)=0$ if $\mu_{z}\left(A_{z, n, i}\right)=0$. By Lemma 3.1 the functions $(y, z) \mapsto \mu_{z, n}^{y}(A)$ are $\sigma(\mathcal{S}(Y)) \otimes \sigma(\mathcal{S}(Z))$-measurable (and Borel measurable if $z \mapsto \mu_{z}$ is Borel), because $I_{A_{z, n, i}}(x)=I_{B_{n, i}}\left(f_{z}(x)\right), I_{A \cap A_{z, n, i}}=I_{A} I_{A_{z, n, i}}$. It is obvious that $\mu_{z, n}^{y}(A)$ coincides with the conditional expectation of the function $I_{A}$ with respect 
to the $\sigma$-algebra $\mathcal{B}_{z, n}$ and the measure $\mu_{z}$. Therefore, for any Borel function $\varphi$ on $X$ the conditional expectation $E_{z}\left(\varphi \mid \mathcal{B}_{z, n}\right)$ of $\varphi$ with respect to $\mathcal{B}_{z, n}$ and $\mu_{z}$ equals

$$
\int_{X} \varphi(x) \mu_{z, n}^{y}(d x)
$$

According to the martingale convergence theorem (see [9, Section 10.3], for every fixed $z$, the constructed functions $E_{z}\left(\varphi \mid \mathcal{B}_{z, n}\right)$ converge $\sigma_{z}$-almost everywhere and in the space $L^{1}\left(\sigma_{z}\right)$ to the conditional expectation $E_{z}\left(\varphi \mid \mathcal{B}_{z}\right)$ of $\varphi$ with respect to the $\sigma$-algebra $\mathcal{B}_{z}$ and the measure $\mu_{z}$.

However, we need conditional measures, not conditional expectations. Of course, it is known that some conditional measures $\mu_{z}^{y}$ exist and define the same conditional expectations. Unfortunately, not every choice of $\mu_{z}^{y}$ is suitable to guarantee the joint measurability in $(y, z)$, because the relations defining conditional expectations hold almost everywhere, not pointwise, and the corresponding measure zero sets depend on $\varphi$ and $z$. So a constructive method of selecting conditional measures is needed.

In order to define our conditional probabilities, we consider the set of points for which the sequence of measures $\mu_{z, n}^{y}$ converges and its limit is concentrated on the set $f_{z}^{-1}(y)$. Convergence is easier achieved on a compact space. By using a countable family in $C_{b}(X)$ separating points, we can embed $X$ continuously into the cube $I:=[0,1]^{\infty}$ and assume that $X$ is a Souslin set in $I$ (equipped with a stronger topology than the one induced from $\left.[0,1]^{\infty}\right)$. The countable family of polynomials in coordinate functions of the form $\sum c_{i_{1}, \ldots, i_{m}, k_{1}, \ldots, k_{m}} x_{i_{1}}^{k_{1}} \cdots x_{i_{m}}^{k_{m}}$, where $c_{i_{1}, \ldots, k_{m}}$ are rational numbers, will be denoted by $\left\{\varphi_{j}\right\}$.

We denote by $\Omega$ the set of all points $(y, z) \in Y \times Z$ for which, for every $\varphi_{j}$, the sequence of integrals

$$
\int_{X} \varphi_{j}(x) \mu_{z, n}^{y}(d x)
$$

has a finite limit as $n \rightarrow \infty$. Every integral is a $\sigma(\mathcal{S}(Y \times Z))$-measurable function of $(y, z)$. Moreover, it is Borel if the mapping $z \mapsto \mu_{z}$ is Borel measurable. Hence $\Omega \in \sigma(\mathcal{S}(Y \times Z))$ and $\Omega \in \mathcal{B}(Y \times Z)$ if $z \mapsto \mu_{z}$ is Borel measurable.

We now use the compactness of $I$, due to which for any $(y, z) \in \Omega$ the sequence of measures $\mu_{z, n}^{y}$ regarded on $X$ converges weakly to a Borel probability measure $\nu_{z}^{y}$ on $I$ (but so far we do not claim that it is concentrated on $X$ ).

According to Lemma 3.3 , the subset

$$
\Omega_{0}:=\left\{(y, z) \in \Omega: \nu_{z}^{y}\left(f_{z}^{-1}(y)\right)=1\right\}
$$

belongs to $\sigma\left(\mathcal{S}(Y \times Z)\right.$ ) (and is Borel if $z \mapsto \mu_{z}$ is Borel). For each $(y, z) \in \Omega_{0}$, the measure $\nu_{z}^{y}$ is obviously concentrated on $X$ (recall that the Souslin set $X$ is measurable with respect to all Borel measures). The set

$$
\Omega_{1}=\left\{(y, z) \in Y \times Z: y \in f_{z}(X)\right\}
$$

is the projection of the graph of $f$ under the mapping

$$
X \times Z \times Y \rightarrow Y \times Z, \quad(x, z, y) \mapsto(y, z) .
$$

This set is Souslin in $Y \times Z$. If each $f_{z}$ is a surjection, then $\Omega_{1}=Y \times Z$.

Now we are going to apply the measurable choice theorem (see [9, Theorem 6.9.2]) to the multivalued mapping $\Psi:(y, z) \mapsto f_{z}^{-1}(y)$ on $\Omega_{1}$ with values in the class of non-empty subsets of $X$. Its graph is the set

$$
\left\{(y, z, u):(y, z) \in \Omega_{1}, u \in f_{z}^{-1}(y)\right\}=\left\{(y, z, u):(y, z) \in \Omega_{1}, f_{z}(u)=y\right\},
$$


which is Souslin, because $(y, z, u) \mapsto f_{z}(u)$ and $(y, z, u) \mapsto y$ are Borel mappings. By the cited theorem there is a mapping

$$
g:(y, z) \mapsto g_{z}(y), \Omega_{1} \rightarrow X
$$

such that

$$
g_{z}(y) \in f_{z}^{-1}(y) \quad \forall z \in Z, y \in f_{z}(X)
$$

and $g$ is measurable with respect to the restriction of $\sigma(\mathcal{S}(Y)) \otimes \sigma(\mathcal{S}(Z))$ to $\Omega_{1}$ and $\mathcal{B}(X)$.

Finally, if $(y, z) \notin \Omega_{0}$ and $y \in f_{z}(X)$, i.e., $(y, z) \in \Omega_{1}$, we set $\nu_{z}^{y}:=\delta_{g_{z}(y)}$, and if $y \notin f_{z}(X)$, we set $\nu_{z}^{y}:=\delta_{x_{0}}$, where $x_{0} \in X$ is a fixed point independent of $y$ and $z$. The constructed family of measures $\nu_{z}^{y}$ is measurable with respect to $\mathcal{S}(Y \times Z)$. Indeed, its restriction to $\Omega_{0}$ is measurable with respect to the trace of $\sigma(\mathcal{S}(Y)) \otimes \sigma(\mathcal{S}(Z))$. The restriction to $\Omega_{1} \backslash \Omega_{0}$ is measurable with respect to the trace of $\sigma(\mathcal{S}(Y \times Z))$, because for every $f \in C_{b}(X)$ the function $f\left(g_{z}(y)\right)$ is $\sigma(\mathcal{S}(Y \times Z))$ measurable by the measurability of $g$. The restriction to the complement of $\Omega_{0} \cup \Omega_{1}$ is constant, and both sets $\Omega_{0}$ and $\Omega_{1}$ are in $\sigma(\mathcal{S}(Y \times Z))$.

It remains to verify that the measures $\nu_{z}^{y}$ serve as conditional probabilities with the required properties. By definition $\nu_{z}^{y}\left(f_{z}^{-1}(y)\right)=1$ for all $y \in Y$ and $z \in Z$. The function

$$
(y, z) \mapsto \int_{X} \varphi_{j}(x) \nu_{z}^{y}(d x)
$$

is $\sigma(\mathcal{S}(Y \times Z))$-measurable for every $\varphi_{j}$, which gives the required measurability of $\left\{\nu_{z}^{y}\right\}$. In order to see that condition 3) from the definition of proper regular conditional measures holds, it is enough to show that, picking arbitrary regular conditional measures $\mu_{z}^{y}$ for $\mu_{z}$ (not necessarily measurable in $z$ ), for every fixed $z \in Z$, we have

$$
\nu_{z}^{y}=\mu_{z}^{y} \quad \text { for } \sigma_{z} \text {-almost every } y .
$$

By the definition of $\sigma_{z}$ this is equivalent to the relation

$$
\nu_{z}^{f(x)}=\mu_{z}^{f(x)} \quad \text { for } \mu_{z} \text {-almost every } x .
$$

This relation holds, since there is a countable family of bounded continuous functions on $X$ separating Borel measures, and for every function $\psi$ from this family its integrals against $\nu_{z}^{f(x)}$ and $\mu_{z}^{f(x)}$ coincide $\mu_{z}$-almost everywhere, because, as explained above, both expressions

$$
\int_{X} \psi(u) \mu_{z}^{f(x)}(d u) \text { and } \int_{X} \psi(u) \nu_{z}^{f(x)}(d u)
$$

serve as the conditional expectation of $\psi$ with respect to $\mathcal{B}_{z}$ and $\mu_{z}$.

Theorem 3.5. Suppose that in Theorem 3.4 for each $z$ the mapping $f_{z}: X \rightarrow Y$ is a Borel surjection possessing a right inverse mapping $g_{z}$ such that $(y, z) \mapsto g_{z}(y)$ is Borel measurable (or, more generally, the set $\bigcup_{z}\left(f_{z}(X) \times\{z\}\right)$ is Borel in $Y \times Z$ and the mapping $(y, z) \mapsto g_{z}(y)$ is Borel measurable); for example, the mapping $f: X \rightarrow Y$ does not depend on $z$ and is a Borel surjection possessing a Borel right inverse mapping $g$. If also $z \mapsto \mu_{z}$ is Borel measurable, then there exists a jointly Borel measurable version of conditional measures $\mu_{z}^{y}$.

In particular, this is true if $X$ is the product of two Souslin spaces $X_{1}$ and $X_{2}$, $f$ is the standard projection onto $X_{2}$, and $z \mapsto \mu^{z}$ is Borel measurable. 
Proof. This follows from our reasoning above (taking into account the notes about Borel measurability), since under stronger assumptions of this theorem we already have jointly Borel measurable right inverse mappings $g_{z}$ without any measurable choice theorems. The corresponding Dirac measures $\delta_{g_{z}(y)}$ defined for all $y, z$ from the complement of the Borel set $\Omega_{0}$ are also jointly Borel measurable and $\Omega_{1}=Y \times Z$ in the surjective case. Similarly we consider the case where $f_{z}$ is not surjective and $\bigcup_{z}\left(f_{z}(X) \times\{z\}\right)$ is Borel.

Note that in the case of the product-space $X=X_{1} \times X_{2}$ and the projection $\pi_{X_{2}}$ on $X_{2}$ it is sometimes more convenient to consider conditional measures on the common space $X_{1}$ in place of the slices $X_{1} \times\left\{x_{2}\right\}=\pi_{X_{2}}^{-1}\left(x_{2}\right) \subset X_{1} \times X_{2}$. Both representations are equivalent and it is easy to pass from one to the other.

The assertion with a single mapping $f$ not depending on the parameter admits an obvious generalization.

Corollary 3.6. Let $X_{1}$ and $X_{2}$ be completely regular Souslin spaces, let $(T, \mathcal{T})$ be a measurable space, and let $t \mapsto \mu_{t}$ be a mapping from $T$ to $\mathcal{P}\left(X_{1} \times X_{2}\right)$ that is measurable with respect to $\mathcal{T}$ and $\mathcal{B}\left(\mathcal{P}\left(X_{1} \times X_{2}\right)\right)$. Then there is a mapping

$$
\left(t, x_{2}\right) \mapsto \mu_{t}^{x_{2}} \in \mathcal{P}\left(X_{1}\right),
$$

measurable with respect to $\mathcal{T} \otimes \mathcal{B}\left(X_{2}\right)$ and $\mathcal{B}\left(\mathcal{P}\left(X_{1}\right)\right)$, such that the measures $\mu_{t}^{x_{2}}$ serve as conditional measures for $\mu_{t}$ and the projection on $X_{2}$.

Proof. The previous theorem can be applied with the space $Z=\mathcal{P}\left(X_{1} \times X_{2}\right)$ as a parameter space, which gives a Borel mapping $\left(\mu, x_{2}\right) \mapsto P_{\mu}^{x_{2}}$ such that $P_{\mu}^{x_{2}}$ serve as conditional measures for $\mu$. Then the mapping $\left(t, x_{2}\right) \mapsto \mu_{t}^{x_{2}}:=P_{\mu_{t}}^{x_{2}}$ is measurable with respect to $\mathcal{T} \otimes \mathcal{B}\left(X_{2}\right)$.

The following parametric version of the so-called gluing lemma (see [42]) has been noted in [32, Theorem 7.3] (for Polish spaces).

Corollary 3.7. Let $X_{1}, X_{2}, X_{3}$ be completely regular Souslin spaces, let $(T, \mathcal{T})$ be a measurable space, and let

$$
t \mapsto \mu_{1,2, t}, T \rightarrow \mathcal{P}\left(X_{1} \times X_{2}\right) \quad \text { and } \quad t \mapsto \mu_{2,3, t}, T \rightarrow \mathcal{P}\left(X_{2} \times X_{3}\right)
$$

be $\mathcal{T}$-measurable mappings such that, for each $t$, the projections of $\mu_{1,2, t}$ and $\mu_{2,3, t}$ on $X_{2}$ coincide. Then there is a $\mathcal{T}$-measurable mapping $t \mapsto \eta_{t}$ from $T$ to the space $\mathcal{P}\left(X_{1} \times X_{2} \times X_{3}\right)$ such that, for each $t$, the projection of $\eta_{t}$ on $X_{1} \times X_{2}$ is $\mu_{1,2, t}$ and the projection on $X_{2} \times X_{3}$ is $\mu_{2,3, t}$.

Proof. It suffices to recall the usual construction of the measure on $X_{1} \times X_{2} \times X_{3}$ for every fixed $t$ via conditional measures (see [12, Lemma 3.3.1] or [41]): using disintegrations

$$
\mu_{1,2, t}\left(d x_{1} d x_{2}\right)=\mu_{1,2, t}^{x_{2}}\left(d x_{1}\right) \pi_{t}\left(d x_{2}\right), \quad \mu_{2,3, t}\left(d x_{2} d x_{3}\right)=\mu_{2,3, t}^{x_{2}}\left(d x_{3}\right) \pi_{t}\left(d x_{2}\right),
$$

where $\pi_{t}$ is the common projection of $\mu_{1,2, t}$ and $\mu_{2,3, t}$ on $X_{2}, \mu_{1,2, t}^{x_{2}}$ and $\mu_{2,3, t}^{x_{2}}$ are the corresponding conditional measures measurably depending on $t$, we set

$$
\eta_{t}\left(d x_{1} d x_{2} d x_{3}\right)=\mu_{1,2, t}^{x_{2}}\left(d x_{1}\right) \mu_{2,3, t}^{x_{2}}\left(d x_{3}\right) \pi_{t}\left(d x_{2}\right) .
$$

This means that for each bounded Borel function $f$ on $X_{1} \times X_{2} \times X_{3}$ we have the following equality:

$$
\int f d \eta_{t}=\int_{X_{2}} \int_{X_{3}} \int_{X_{1}} f\left(x_{1}, x_{2}, x_{3}\right) \mu_{1,2, t}^{x_{2}}\left(d x_{1}\right) \mu_{2,3, t}^{x_{2}}\left(d x_{3}\right) \pi_{t}\left(d x_{2}\right) .
$$


The measurability of the mapping $t \mapsto \eta_{t}$ follows by the measurability of conditional measures and the projection. The fact that $\eta_{t}$ has the prescribed projections is verified directly (see [12, Lemma 3.3.1]).

Remark 3.8. The assumption that the space of parameters $Z$ is Souslin is quite natural in the situation of Theorem [3.4. However, in the situation of Theorem [3.5] for $Z$ we can take an arbitrary measurable space $(Z, \mathcal{Z})$ without any topology. The same reasoning shows that if $(z, x) \mapsto f_{z}(x)$ is $\mathcal{Z} \otimes \mathcal{B}(X)$-measurable, each $f_{z}$ is a surjection that admits a right inverse mapping $g_{z}$ for which $(z, y) \mapsto g_{z}(y)$ is $\mathcal{Z} \otimes \mathcal{B}(Y)$-measurable, and $\mu_{z}$ is $\mathcal{Z}$-measurable, then there are conditional measures $\mu_{z}^{y}$, measurable with respect to $\mathcal{Z} \otimes \mathcal{B}(Y)$.

Remark 3.9. (i) It is known that the $\sigma$-algebra $\sigma(\mathcal{S}(X))$ is not countably generated for any uncountable Polish space $X$ (see [9, Example 6.5.9]), unlike the Borel $\sigma$ algebra. This is one of the reasons why the Borel measurability can be preferable in applications.

(ii) One can show that if $Y$ and $Z$ are uncountable Souslin spaces, then the product $\sigma$-algebra $\sigma(\mathcal{S}(Y) \otimes \mathcal{S}(Z))$ is strictly smaller than the $\sigma$-algebra $\sigma(\mathcal{S}(Y \times Z))$ of the product-space.

(iii) The existence of conditional expectations measurable with respect to a parameter can be obtained under broader assumptions, because in this case there is no problem with property 1 ) of conditional measures. The continuity of conditional expectations with respect to a parameter was studied in [26].

We now see that the existence of jointly Borel conditional measures depending on the parameter $z$ implies some restrictions on the mappings $f_{z}$, so that such joint Borel measurability cannot be always guaranteed. The next proposition is a parametrized version of the known result of Blackwell and Ryll-Nardzewski [8] for single measures.

Proposition 3.10. Let $X, Y, Z$ be Polish spaces. Suppose that there is a jointly Borel measurable version of conditional measures $\mu_{z}^{y}$ concentrated on the sets $f_{z}^{-1}(y)$ for all $y \in Y$ and $z \in Z$. Then there is a Borel mapping $g: Z \times Y \rightarrow X$ such that $f_{z}(g(z, y))=y$ for all $y \in Y$ and $z \in Z$.

Proof. We shall use the following result of Blackwell and Ryll-Nardzewski [8] (see also [29, Corollary 18.7] or [9, Exercise 10.10.47], where the hint contains the proof). For our convenience we change their notation of spaces. Suppose that $U$ and $X$ are Borel sets in Polish spaces, $\mathcal{A}$ is a countably generated sub- $\sigma$-algebra in $\mathcal{B}(U)$ and for each $u \in U$ there is a measure $\mu^{u} \in \mathcal{P}(X)$ such that the function $u \mapsto \mu^{u}(B)$ is $\mathcal{A}$-measurable for every set $B \in \mathcal{B}(X)$. Let $S \subset X \times U$ be a set such that $\mu^{u}\left(S_{u}\right)>0$ for all $u \in U$, where $S_{u}=\{x \in X:(x, u) \in S\}$. Then $S$ contains the graph of an $(\mathcal{A}, \mathcal{B}(X))$-measurable mapping $F: U \rightarrow X$.

We apply this result in the situation where $U=Z \times X, \mathcal{A}$ is the sub- $\sigma$-algebra in $\mathcal{B}(Z \times X)$ generated by the mapping

$$
\begin{gathered}
h: Z \times X \rightarrow Z \times Y, \quad(z, x) \mapsto\left(z, f_{z}(x)\right), \\
\mu^{u}=\mu_{z}^{f_{z}(x)}, \quad u=(z, x),
\end{gathered}
$$

and

$$
S=\left\{(z, x, v) \in Z \times X \times X: f_{z}(v)=f_{z}(x)\right\}
$$


The section $S_{u}$ is defined by

$$
S_{u}=S_{z, x}=\left\{v \in X: f_{z}(v)=f_{z}(x)\right\}=f_{z}^{-1}\left(f_{z}(x)\right),
$$

hence $\mu^{u}\left(S_{u}\right)=\mu_{z}^{f_{z}(x)}\left(f_{z}^{-1}\left(f_{z}(x)\right)\right)=1$. By the cited result there is a mapping $F: Z \times X \rightarrow X$ with the graph in $S$ such that $F$ is $\mathcal{A}$-measurable. The latter means that there is a Borel mapping $g: Z \times Y \rightarrow X$ such that $F(z, x)=g(h(z, x))$. Since the graph of $F$ is contained in $S$, by the definition of $h$ we obtain

$$
f_{z}\left(g\left(z, f_{z}(x)\right)\right)=f_{z}(x) \quad \forall x \in X, z \in Z .
$$

It follows that $f_{z}(g(z, y))=y$ for all $z \in Z$ and $y \in Y$.

It is known that in general there is no $g$ with the stated properties (see, e.g., [9, §6.9]). A sufficient condition for the existence of $g$ is this: for each $y \in Y$ and $z \in Z$ the set $f_{z}^{-1}(y)$ is a countable union of compact sets. Indeed, we consider again the Borel mapping $h:(z, x) \mapsto\left(z, f_{z}(x)\right)$ and observe that the sets $h^{-1}(z, y)$ are countable unions of compact sets. Hence by a classical result (see Theorem $\mathrm{C}$ in the next section) there is a Borel mapping $g: Z \times Y \rightarrow X$ with the graph contained in the set $\left\{(z, y, x): f_{z}(x)=y\right\}$.

\section{Kantorovich Problems With a PARAmeter}

We now turn to Kantorovich optimal plans depending on a parameter.

Let $X$ and $Y$ be completely regular Souslin spaces (for example, Polish spaces). The corresponding spaces of Borel probability measures $\mathcal{P}(X)$ and $\mathcal{P}(Y)$ will be equipped with their weak topologies (making them Souslin or Polish spaces, respectively). By $\pi_{X}$ and $\pi_{Y}$ we denote the projections of $X \times Y$ on $X$ and $Y$.

For any pair of measures $\mu \in \mathcal{P}(X)$ and $\nu \in \mathcal{P}(Y)$, the set

$$
\Pi(\mu, \nu)=\left\{\sigma \in \mathcal{P}(X \times Y): \sigma \circ \pi_{X}^{-1}=\mu, \sigma \circ \pi_{Y}^{-1}=\nu\right\}
$$

is convex and compact in the weak topology, which follows from Prohorov's theorem. This set is not empty: it always contains the product of $\mu$ and $\nu$.

Recall that a function $f$ is lower semicontinuous if the sets $\{f \leq c\}$ are closed. It is known (see [12, Corollary 4.3.4]) that if $f$ is a bounded lower semicontinuous function on $X$ and Borel probability measures $\mu_{n}$ on $X$ converge weakly to $\mu$, then

$$
\int_{X} f d \mu \leq \liminf _{n \rightarrow \infty} \int_{X} f d \mu_{n}
$$

Given a lower semicontinuous cost function $h \geq 0$ on $X \times Y$ and a pair of measures $\mu \in \mathcal{P}(X)$ and $\nu \in \mathcal{P}(Y)$, in the aforementioned Kantorovich problem of finding the infimum of $K_{h}(\mu, \nu)$ of the quantity $I_{h}(\sigma)$ over all measures $h \in \Pi(\mu, \nu)$ the minimum is attained if there is a measure $\sigma$ with $I_{h}(\sigma)<\infty$ (which is always true if $h$ is bounded).

Let $(T, \mathcal{T})$ be a measurable space. In the case where $T$ is a topological space we assume that $\mathcal{T}$ is its Borel $\sigma$-algebra $\mathcal{B}(T)$.

Assume also that

$$
h: T \times X \times Y \rightarrow[0,+\infty)
$$

is a $\mathcal{T} \otimes \mathcal{B}(X) \otimes \mathcal{B}(Y)$-measurable function such that $h_{t}:(x, y) \mapsto h(t, x, y)$ is lower semicontinuous for each $t$.

Thus, we obtain a Kantorovich problem with a parameter. Dependence on a parameter appears even for a single cost function if marginal distributions depend on $t$. We consider the case where both marginals and the cost function depend on $t$. 
Let $t \mapsto \mu_{t}, T \rightarrow \mathcal{P}(X)$ be a $(\mathcal{T}, \mathcal{B}(\mathcal{P}(X)))$-measurable mapping and let $t \mapsto \nu_{t}$, $T \rightarrow \mathcal{P}(Y)$ be a $(\mathcal{T}, \mathcal{B}(\mathcal{P}(Y)))$-measurable mapping.

Theorem 4.1. Suppose that the transportation costs $K(t):=K_{h_{t}}\left(\mu_{t}, \nu_{t}\right)$ are finite and the cost functions $h_{t}:(x, y) \mapsto h(t, x, y)$ are continuous. Then the function $K$ is $(\mathcal{T}, \mathcal{B}(\mathcal{P}(X \times Y)))$-measurable. In addition, one can choose optimal measures $\sigma_{t}$ such that the mapping $t \mapsto \sigma_{t}$ is measurable with respect to $\sigma(\mathcal{S}(\mathcal{T}))$ and $\mathcal{B}(\mathcal{P}(X \times Y))$.

In the next theorem we remove the assumption of continuity of cost functions and reinforce the conclusion by the existence of Borel measurable selections, but $T$ is required to be a Souslin space.

Theorem 4.2. Suppose that $T$ is a Souslin space, $t \mapsto \mu_{t}$ and $t \mapsto \nu_{t}$ are Borel mappings with values in the spaces $\mathcal{P}(X)$ and $\mathcal{P}(Y)$, respectively, and the corresponding transportation costs $K_{h_{t}}\left(\mu_{t}, \nu_{t}\right)$ are finite. Then the function $t \mapsto K_{h_{t}}\left(\mu_{t}, \nu_{t}\right)$ is Borel measurable and there is a mapping $t \mapsto \sigma_{t}, T \rightarrow \mathcal{P}(X \times Y)$, measurable with respect to $\mathcal{B}(T)$ and $\mathcal{B}(\mathcal{P}(X \times Y))$, such that for all $t \in T$ we have

$$
\sigma_{t} \in \Pi\left(\mu_{t}, \nu_{t}\right), \quad \int h(t, x, y) \sigma_{t}(d x d y)=K_{h_{t}}\left(\mu_{t}, \nu_{t}\right)
$$

Corollary 4.3. In the previous theorem, there is a sequence of Borel measurable mappings $\Phi_{n}: T \rightarrow \mathcal{P}(X \times Y)$ such that, for every $t \in T$, the sequence $\left\{\Phi_{n}(t)\right\}$ is dense in the convex compact set $M_{t}$ of $h_{t}$-optimal measures in $\Pi\left(\mu_{t}, \nu_{t}\right)$.

Corollary 4.4. In the previous theorem, the optimal plans $\sigma_{t}$ admit disintegrations

$$
\sigma_{t}=\int_{Y} \sigma_{t}^{y} \nu_{t}(d y)
$$

with Borel probability measures $\sigma_{t}^{y}$ on $X$ that are Borel measurable in $(t, y)$.

For Souslin spaces $X$ and $Y$ we have the following result.

Theorem 4.5. Let $X$ and $Y$ be completely regular Souslin spaces and let $T$ be a Souslin space. Let $(x, y) \mapsto h(t, x, y)$ be continuous for every $t$ and let $t \mapsto \mu_{t}$ and $t \mapsto \nu_{t}$ be Borel measurable. Then the function $t \mapsto K(t)$ is measurable with respect to $\sigma(\mathcal{S}(T))$.

Note that if in the last theorem the function $t \mapsto K(t)$ is Borel measurable, then there is a sequence of mappings $\Phi_{n}: T \rightarrow \mathcal{P}(X \times Y)$, measurable with respect to $(\sigma(\mathcal{S}(T)), \mathcal{B}(\mathcal{P}(X \times Y)))$, such that, for every $t \in T$, the sequence $\left\{\Phi_{n}(t)\right\}$ is dense in the convex compact set $M_{t}$ of $h_{t}$-optimal measures in $\Pi\left(\mu_{t}, \nu_{t}\right)$.

\section{Auxiliary Results and proofs}

The following general version of the Kantorovich duality for finite nonnegative lower semicontinuous cost functions holds:

$$
\begin{aligned}
& K_{h}(\mu, \nu)=\sup \left\{\int \varphi d \mu+\int \psi d \nu:\right. \\
& \left.\qquad \varphi \in C_{b}(X), \psi \in C_{b}(Y), \varphi(x)+\psi(y) \leq h(x, y)\right\} .
\end{aligned}
$$


See [5], 6], [30], 35], and [42] for a discussion of this duality; a short derivation of the general case from the case of bounded continuous cost functions is given in [6]. Hence for each $\varepsilon>0$ there are functions $\varphi \in C_{b}(X)$ and $\psi \in C_{b}(Y)$ such that

$$
\varphi(x)+\psi(y) \leq h(x, y) \text { for all } x \text { and } y
$$

and

$$
K_{h}(\mu, \nu) \leq \int \varphi d \mu+\int \psi d \nu+\varepsilon
$$

Moreover, for bounded $h$, in the right-hand side of (5.1) one can take the supremum over $\varphi$ and $\psi$ such that $|\varphi| \leq\|h\|_{\infty},|\psi| \leq\|h\|_{\infty}$. This is explained in [42, Remark 1.13], but for the reader's convenience we give a straightforward justification. We can assume that $\|h\|_{\infty}=1$. If a pair $\varphi, \psi$ satisfies the indicated bound, then, for any number $t$, the pair $\varphi+t, \psi-t$ also satisfies this bound and the sum of the corresponding integrals is the same. Hence we can assume that $\sup _{x} \varphi(x)=1$. Hence $\psi(y) \leq 0$. Next, we replace $\varphi$ by $\varphi_{1}=\max (\varphi, 0)$ and obtain a pair with $\varphi_{1}(x)+\psi(y) \leq h(x, y)$ and $0 \leq \varphi_{1} \leq 1$ for which the integral of $\varphi_{1}$ is not less than that of $\varphi$. Finally, we replace $\psi$ by $\psi_{1}=\max (\psi,-1)$, which keeps the upper bound by $h$ and increases the integral. Hence we obtain a pair with $0 \leq \varphi_{1} \leq 1$, $-1 \leq \psi_{1} \leq 0$ and $\varphi_{1}(x)+\psi_{1}(y) \leq h(x, y)$ for which the sum of the respective integrals dominates the original sum. The next lemma is an immediate corollary of this bound.

Lemma 5.1. Let $h \leq 1$. Then for all $\mu_{1}, \mu_{2} \in \mathcal{P}(X)$ and $\nu_{1}, \nu_{2} \in \mathcal{P}(Y)$ we have

$$
\left|K_{h}\left(\mu_{1}, \nu_{1}\right)-K_{h}\left(\mu_{2}, \nu_{2}\right)\right| \leq\left\|\mu_{1}-\mu_{2}\right\|+\left\|\nu_{1}-\nu_{2}\right\| \text {. }
$$

Proof. We can assume that $K_{h}\left(\mu_{1}, \nu_{1}\right)>K_{h}\left(\mu_{2}, \nu_{2}\right)$. Let $\varepsilon>0$. There are functions $\varphi \in C_{b}(X), \psi \in C_{b}(Y)$ with $\varphi(x)+\psi(y) \leq h(x, y),|\varphi| \leq 1,|\psi| \leq 1$ such that

$$
K_{h}\left(\mu_{1}, \nu_{1}\right)<\int \varphi d \mu_{1}+\int \psi d \nu_{1}+\varepsilon
$$

Since

$$
K_{h}\left(\mu_{2}, \nu_{2}\right) \geq \int \varphi d \mu_{2}+\int \psi d \nu_{2}
$$

we have

$$
K_{h}\left(\mu_{1}, \nu_{1}\right)-K_{h}\left(\mu_{2}, \nu_{2}\right) \leq \varepsilon+\int \varphi d\left(\mu_{1}-\mu_{2}\right)+\int \psi d\left(\nu_{1}-\nu_{2}\right)
$$

whence our claim follows with the extra term $\varepsilon$ on the right, so it remains to let $\varepsilon \rightarrow 0$.

Lemma 5.2. Let $(T, \mathcal{T})$ be a measurable space, $Z$ a Polish space, and let $t \mapsto \mu_{t}$ be a mapping from $T$ to $\mathcal{P}(Z)$ measurable with respect to $\mathcal{T}$ and $\mathcal{B}(\mathcal{P}(Z))$. Then there is a sequence of increasing compact sets $Z_{n}(t) \subset Z$ such that the sets $\bigcup_{t}\left(\{t\} \times Z_{n}(t)\right)$ are in $\mathcal{T} \otimes \mathcal{B}(Z)$, the set-valued mapping $t \mapsto Z_{n}(t)$ is $\mathcal{T}$-measurable, the normalized restrictions $\mu_{t}^{n}$ of $\mu_{t}$ to $Z_{n}(t)$ define mappings $t \mapsto \mu_{t}^{n}$ from $T$ to $\mathcal{P}(Z)$ measurable in the same sense and $\left\|\mu_{t}^{n}-\mu_{t}\right\| \rightarrow 0$.

The same is true if $Z$ is a completely regular Luzin space, hence this is true if $Z$ is a Borel set in a Polish space.

Proof. It suffices to introduce the parameter $t$ in the standard proof of Ulam's theorem. We consider $Z$ with a complete separable metric and take a dense countable 
set $\left\{z_{j}\right\} \subset Z$. Let $n \in \mathbb{N}$. For each $k$ and $m$ in $\mathbb{N}$ let $A_{k, m}$ be the union of $m$ closed balls of radius $2^{-k}$ centered at $z_{1}, \ldots, z_{m}$. Then $\mu_{t}\left(A_{k, m}\right) \rightarrow 1$ as $m \rightarrow \infty$. Let

$$
\begin{gathered}
N_{n, k}(t)=\min \left\{m: \mu_{t}\left(A_{k, m}\right)>1-2^{-n-k}\right\}, \\
Z_{n}(t)=\bigcap_{k \geq 1} A_{k, N_{n, k}}(t) .
\end{gathered}
$$

The sets $A_{k, N_{n, k}}(t)$ are closed. Hence the sets $Z_{n}(t)$ are also closed. In addition, each $Z_{n}(t)$ is contained in finitely many balls of radius $2^{-k}$ for each $k$. Hence $Z_{n}(t)$ is compact. By construction,

$$
\mu_{t}\left(X \backslash Z_{n}(t)\right)<\sum_{k=1}^{\infty} 2^{-n-k}=2^{-n} .
$$

Let $\mu_{t}^{n}$ be the normalized restriction of $\mu_{t}$ to $Z_{n}(t)$. Then $\left\|\mu_{t}-\mu_{t}^{n}\right\|<2^{-n}\left(1-2^{-n}\right)^{-1}$. We have $Z_{n}(t) \subset Z_{n+1}(t)$, since $N_{n, k}(t) \leq N_{n, k+1}(t)$, so $A_{k, N_{n, k}}(t) \subset A_{k, N_{n, k+1}}(t)$.

The functions $t \mapsto N_{n, k}(t)$ are $\mathcal{T}$-measurable, since the set $N_{n, k}^{-1}(q)$ is the intersection of the sets $\left\{t: \mu_{t}\left(A_{k, j}\right) \leq 1-2^{-n-k}\right\}$ with $j<q$ and $\left\{t: \mu_{t}\left(A_{k, q}\right)>1-2^{-n-k}\right\}$ that are $\mathcal{T}$-measurable, which readily follows from the measurability of $t \mapsto \mu_{t}$. In order to show the measurability of $\mu_{t}^{n}$ it suffices to show the measurability of the mapping $\left.t \mapsto \mu\right|_{N_{n}(t)}$. This mapping is the limit of restrictions of $\mu_{t}$ to the decreasing sets $\bigcap_{k=1}^{m} A_{k, N_{n, k}}(t)$. Such restrictions $\mu_{t}^{n, m}$ are $\mathcal{T}$-measurable. Indeed, the sets $N_{n, k}^{-1}(q)$ are $\mathcal{T}$-measurable, hence so are their finite intersections, but $\mu_{t}^{n, m}$ has countably many values assumed on such intersections.

Every set $\bigcup_{t}\left(\{t\} \times Z_{n}(t)\right)$ belongs to $\mathcal{T} \otimes \mathcal{B}(Z)$, because it is the intersection of the sets $\bigcup_{t}\left(\{t\} \times \bigcap_{k=1}^{m} A_{k, N_{n, k}}(t)\right)$, which are in $\mathcal{T} \otimes \mathcal{B}(Z)$, since they are countable unions of sets of the form $T_{k, n, m} \times A_{k, m}$ with $T_{k, n, m}=\left\{t: N_{k, n}(t)=m\right\}$. Let us show that the set-valued mapping $t \mapsto Z_{n}(t)$ is $\mathcal{T}$-measurable. It suffices to show that for every $x \in X$ the real function $t \mapsto \operatorname{dist}\left(x, Z_{n}(t)\right)$ is $\mathcal{T}$-measurable, see [17, Theorem III.9] or [3, Chapter 8]. Let $D_{n, m}(t)=\bigcap_{k=1}^{m} A_{k, N_{n, k}}(t)$. We observe that

$$
d_{H}\left(Z_{n}(t), D_{n, m}(t)\right) \rightarrow 0 \quad \text { and } \quad \operatorname{dist}\left(x, D_{n, m}(t)\right) \rightarrow \operatorname{dist}\left(x, Z_{n}(t)\right) \quad \text { as } m \rightarrow \infty \text {. }
$$

Indeed, for every fixed $\varepsilon>0$ there is $m$ such that $D_{n, m}(t)$ is contained in the $\varepsilon$ neighborhood of $Z_{n}(t)$, because otherwise there is a sequence of points $x_{m} \in D_{n, m}(t)$ with $\operatorname{dist}\left(x_{m}, Z_{n}(t)\right) \geq \varepsilon$. Each $D_{n, m}(t)$ is a union of finitely many balls of radius $2^{-k}$, hence $\left\{x_{m}\right\}$ is precompact and has a limit point $x_{0}$. This point must belong to all $D_{n, m}(t)$, hence to $Z_{n}(t)$, which is impossible, $\operatorname{since} \operatorname{dist}\left(x_{0}, Z_{n}(t)\right) \geq \varepsilon$. This proves the first relation, the second is its corollary.

The case of Luzin spaces follows from the considered case, because $Z$ admits a stronger Polish topology that generates a stronger Polish topology on $\mathcal{P}(Z)$ with the same Borel sets as in the original topology, so the measurability of $\mathcal{P}(Z)$-valued mappings remains the same. Finally, we recall that any Borel set in a Polish space is the image of a Polish space under a continuous injective mapping (see [9, Corollary 6.8.5]).

Remark 5.3. Under a stronger condition that $t \mapsto \mu_{t}(A)$ is $\mathcal{T}$-measurable for every Souslin set $A$ (which does not follow automatically) the previous assertion extends to the case of a Souslin subspace $Z$ in a Polish space $E$ and gives increasing compact sets $Z_{n}(t)$ such that the functions $(t, x) \mapsto I_{Z_{n}(t)}(x)$ are $\mathcal{T} \otimes \sigma(\mathcal{S}(Z))$-measurable and $\mu_{t}\left(Z_{n}(t)\right)>1-2^{-n}$. To this end, we first take such compact sets $Z_{n}^{1}(t)$ in $E$ 
and then consider a parametric version of the standard proof of measurability of sets obtained by means of the Souslin operation (see [9, Theorem 1.10.5]). Recall that $Z$ can be written as

$$
Z=\bigcup_{\left(n_{i}\right)} \bigcap_{k=1}^{\infty} E_{n_{1}, \ldots, n_{k}},
$$

where $\left\{E_{n_{1}, \ldots, n_{k}}\right\}$ is a certain monotone table of closed balls of rational radii centered at points of a fixed countable dense set and the union is taken over all natural sequences $\left(n_{i}\right)$. For every collection $m_{1}, \ldots, m_{k}$ of natural numbers, denote by $D_{m_{1}, \ldots, m_{k}}$ the union of the sets $E_{n_{1}, \ldots, n_{k}}$ over all $n_{1} \leq m_{1}, \ldots, n_{k} \leq m_{k}$. This is a closed set. It is clear from the proof of the cited theorem (taking into account Remark 3.2) that one can find numbers $m_{k}(t)$ measurably depending on $t$ such that

$$
\mu_{t}\left(D_{m_{1}(t), \ldots, m_{k}(t)} \cap Z_{n}^{1}(t)\right)>1-2^{-n} .
$$

Then

$$
\mu_{t}\left(\bigcap_{k=1}^{\infty} D_{m_{1}(t), \ldots, m_{k}(t)} \cap Z_{n}^{1}(t)\right) \geq 1-2^{-n} .
$$

It is verified in that proof that $\bigcap_{k=1}^{\infty} D_{m_{1}(t), \ldots, m_{k}(t)}$ is contained in $Z$. It is clear that this set is closed, so its intersection with $Z_{n}^{1}(t)$ is compact.

Lemma 5.4. Suppose that $(T, \mathcal{T})$ is a measurable space, $X$ and $Y$ are Polish (or Luzin) spaces, $t \mapsto \mu_{t}$ and $t \mapsto \nu_{t}$ are $\mathcal{T}$-measurable mappings with values in $\mathcal{P}(X)$ and $\mathcal{P}(Y)$, correspondingly. Let $(x, y) \mapsto h(t, x, y)$ be lower semicontinuous and $K_{h_{t}}\left(\mu_{t}, \nu_{t}\right)<\infty$ for each $t$. Then for the measures $\mu_{t}^{n}$ and $\nu_{t}^{n}$ from the previous lemma applied to $\mu_{t}$ and $\nu_{t}$ we have

$$
K_{h_{t}}\left(\mu_{t}, \nu_{t}\right)=\lim _{n \rightarrow \infty} K_{h_{t}}\left(\mu_{t}^{n}, \nu_{t}^{n}\right) \quad \forall t \in T .
$$

Proof. Let $t$ be fixed. We have

$$
\mu_{t}^{n} \leq p_{n}(t) \mu_{t}^{n+1} \quad \text { and } \quad \mu_{t}^{n} \leq q_{n}(t) \mu_{t}
$$

where $q_{n}(t)>1$ and $p_{n}(t)>1$ are numbers converging to 1 . Hence there is a finite limit $\lim _{n \rightarrow \infty} K_{h_{t}}\left(\mu_{t}^{n}, \nu_{t}^{n}\right) \leq K_{h_{t}}\left(\mu_{t}, \nu_{t}\right)$. We now prove the opposite inequality. Let $\sigma_{t}^{n} \in \Pi\left(\mu_{t}^{n}, \nu_{t}^{n}\right)$ be optimal measures for $h_{t}$. Both sequences $\left\{\mu_{t}^{n}\right\}$ and $\left\{\nu_{t}^{n}\right\}$ are uniformly tight, hence $\left\{\sigma_{t}^{n}\right\}$ is also uniformly tight and contains a weakly convergent subsequence, which we denote by the same indices. Let $\sigma_{t}$ be its limit. Clearly, $\sigma_{t} \in \Pi\left(\mu_{t}, \nu_{t}\right)$. The integral of $h_{t}$ against $\sigma_{t}$ does not exceed the liminf of the integrals of $h_{t}$ against the measures $\sigma_{t}^{n}$ (see [12, Corollary 4.3.4]), which is exactly the limit of $K_{h_{t}}\left(\mu_{t}^{n}, \nu_{t}^{n}\right)$.

Let us recall the following classical result going back to Novikoff and Kunugui, see [19, p. 224, 225] (or [29, Theorem 18.18], where $X$ is a standard Borel space).

Theorem A. Let $X$ be a Souslin space, $Y$ a Polish space, and $B \subset X \times Y$ a Borel set such that for all $x \in X$ the sections $B_{x}$ are $\sigma$-compact (countable unions of compact sets). Then $B$ admits a Borel uniformization, which means that the projection $\pi_{X}(B)$ of $B$ on $X$ is a Borel set and there is a Borel mapping

$$
f: \pi_{X}(B) \rightarrow Y
$$

whose graph is contained in B. 
There is also another classical result with somewhat different assumptions (see, e.g., [9, Theorem 6.9.3 and Corollary 6.9.4]).

Theorem B. Let $(T, \mathcal{T})$ be a general measurable space, let $E$ be a Polish space, and let $\Psi$ be a mapping on $T$ with values in the set of nonempty closed subsets of $E$ that is measurable in the following sense: for every open set $U \subset E$, the projection of the set $\{(t, x): x \in \Psi(t) \cap U\}$ on $T$ belongs to $\mathcal{T}$. Then there is a $(\mathcal{T}, \mathcal{B}(E))$ measurable mapping $\zeta: T \rightarrow E$ with $\zeta(t) \in \Psi(t)$ for all $t$, i.e., a $(\mathcal{T}, \mathcal{B}(E))$ measurable selection. Moreover, there is a sequence of $(\mathcal{T}, \mathcal{B}(E))$-measurable mappings $\zeta_{n}: T \rightarrow X$ such that the sequence $\left\{\zeta_{n}(t)\right\}$ is dense in $\Psi(t)$ for each $t$.

The difference between the two theorems is that in the latter the space $T$ is more general, but the hypotheses include the measurability of the aforementioned projections, while in the former this measurability follows from other assumptions (here we consider $\Psi(x)=B_{x}$ in order to compare the settings). Indeed, to see this we observe that it suffices to verify the required measurability for closed sets $U$ (since any open set in a Polish space is some countable union of closed sets). But then the sections of $B \cap(X \times U)$ remain $\sigma$-compact, so the projection remains Borel. Note that in Theorem A there is also a sequence of Borel mappings $f_{n}: \pi_{X}(B) \rightarrow Y$ such that $\left\{f_{n}(x)\right\}$ is dense in $B_{x}$ for each $x \in \pi_{X}(B)$.

Thus, Theorem B is formally more general (but to see this we need Theorem A), however, practically the most difficult part is to verify the measurability of projections (and the proof of Theorem $\mathrm{A}$ is more difficult). So our main tool will be Theorem A. It should be noted that Theorem A is not valid for arbitrary measurable spaces in place of Souslin spaces (it fails even for co-analytic sets in $[0,1]$ and single-valued sections).

Finally, let us mention yet another known result (see [9, Theorem 6.9.5]) in which the assumptions are weaker, but also the guaranteed measurability of selections is weaker.

Theorem C. Suppose that $T$ and $E$ are Souslin spaces. Let $\Psi$ be a multivalued mapping from $T$ to the set of nonempty subsets of $E$ such that its graph

$$
\Gamma_{\Psi}=\{(t, u): t \in T u \in \Psi(t)\}
$$

is a Souslin set in $T \times E$. Then, there exists a sequence of selections $\zeta_{n}$ that are measurable as mappings from $(T, \sigma(\mathcal{S}(T)))$ to $(E, \mathcal{B}(E))$ and, for every $t \in T$, the sequence $\left\{\zeta_{n}(t)\right\}$ is dense in the set $\Psi(t)$.

In our situation, a typical application of these results is this.

The set-valued mapping

$$
(\mu, \nu) \mapsto \Pi(\mu, \nu)
$$

from $\mathcal{P}(X) \times \mathcal{P}(Y)$ to the set of nonempty compact subsets of $\mathcal{P}(X \times Y)$ is measurable in the aforementioned sense. Alternatively, we can apply Theorem A by using the easy fact that the set $B$ of triples $(\mu, \nu, \sigma)$ in $\mathcal{P}(X) \times \mathcal{P}(Y) \times \mathcal{P}(X \times Y)$ such that $\sigma \circ \pi_{X}^{-1}=\mu$ and $\sigma \circ \pi_{Y}^{-1}=\nu$ is Borel and its sections $B_{\mu, \nu}$ are compact. Hence there is a sequence of Borel mappings

$$
\Phi_{n}: \mathcal{P}(X) \times \mathcal{P}(Y) \rightarrow \mathcal{P}(X \times Y)
$$

such that the sequence $\left\{\Phi_{n}(\mu, \nu)\right\}$ is dense in $\Pi(\mu, \nu)$ for all $\mu$ and $\nu$.

Let $t \mapsto \mu_{t}$ and $t \mapsto \nu_{t}$ be measurable mappings from $(T, \mathcal{T})$ to the spaces $\mathcal{P}(X)$ and $\mathcal{P}(Y)$ of Borel probability measures on Polish spaces $X$ and $Y$. Then there is a 
sequence of measurable mappings $\Psi_{n}: T \rightarrow \mathcal{P}(X \times Y)$ such that $\Psi_{n}(t) \in \Pi\left(\mu_{t}, \nu_{t}\right)$ and the sequence $\left\{\Psi_{n}(t)\right\}$ is dense in $\Pi\left(\mu_{t}, \nu_{t}\right)$ for each $t$. To this end, we set $\Psi_{n}(t):=\Phi_{n}\left(\mu_{t}, \nu_{t}\right)$.

Suppose now that $(x, y) \mapsto h(t, x, y)$ is continuous for each fixed $t \in T$ (the case of Theorem 4.1). Then the function

$$
K(t)=K_{h_{t}}\left(\mu_{t}, \nu_{t}\right)
$$

is measurable on $T$, which proves the first assertion of Theorem 4.1. Indeed,

$$
K(t)=\inf _{n} \int_{X \times Y} h(t, x, y) \Psi_{n}(t)(d x d y) .
$$

Let now

$$
M_{t}:=\left\{\sigma \in \Pi\left(\mu_{t}, \nu_{t}\right): \int h(t, x, y) \sigma(d x d y)=K(t)\right\} .
$$

Each set $M_{t}$ is compact in $\Pi\left(\mu_{t}, \nu_{t}\right) \subset \mathcal{P}(X \times Y)$. Once we have the measurability of the set-valued mapping $t \mapsto M_{t}$ we can use selection theorems. However, the problem is to verify this measurability. This will be done below for Souslin spaces $T$ in order to have the Borel measurability. However, if we are satisfied with the measurability with respect to the $\sigma$-algebra $\sigma(\mathcal{S}(\mathcal{T}))$ on $T$, then we can apply Theorem B to this larger $\sigma$-algebra. The hypothesis of Theorem $\mathrm{B}$ is fulfilled. Indeed, let $U$ be an open set in $\mathcal{P}(X \times Y)$. The set of pairs $(t, \sigma)$ in $T \times \mathcal{P}(X \times Y)$, where $\sigma \in \Pi\left(\mu_{t}, \nu_{t}\right)$ and the integral of $h_{t}$ against $\sigma$ is $\mathcal{T}$-measurable, is contained in $\mathcal{T} \otimes \mathcal{B}(\mathcal{P}(X \times Y))$ by the $\mathcal{T}$-measurability of $K$. Hence the intersection of this set with $T \times U$ is also in $\mathcal{T} \otimes \mathcal{B}(\mathcal{P}(X \times Y))$. Therefore, the projection of this intersection belongs to $\mathcal{S}(\mathcal{T})$ by a known result (see [9, Corollary 6.10.10]).

Finally, the proof of Theorem 4.5 is completely analogous, the only difference is that now we apply Theorem $\mathrm{C}$ : the set of pairs $(t, \sigma)$ in $T \times \mathcal{P}(X \times Y)$ such that $\sigma \in \Pi_{t}\left(\mu_{t}, \nu_{t}\right)$ is Borel as above. Hence there is a sequence of $\mathcal{S}(T)$-measurable mappings $\Psi_{n}: T \rightarrow \mathcal{P}(X \times Y)$ such that the sequence $\left\{\Psi_{n}(t)\right\}$ is dense in $\Pi_{t}\left(\mu_{t}, \nu_{t}\right)$, so $K(t)$ equals the infimum of the sequence of integrals of $h_{t}$ against $\Psi_{n}(t)$. Once we know that $K(t)$ is Borel measurable, the same reasoning applies to the set of pairs $(t, \sigma)$ with the additional restriction that the integral of $h_{t}$ against $\sigma$ equals $K(t)$, but this restriction determines a Borel set.

Lemma 5.5. Suppose that $Z$ is a Borel set in a complete separable metric space with a metric d, $T$ is a Souslin space, and $h: T \times Z \rightarrow[0,2]$ is a Borel function that is lower semicontinuous in the second variable and has the following property: for every $t$ there is a compact set $Z_{t} \subset Z$ such that $h(t, z) \in[0,1)$ for all $z \in Z_{t}$ and $h(t, z)=2$ for all $z \in Z \backslash Z_{t}$. Then there is a sequence of Borel mappings $\psi_{j}: T \rightarrow Z$ such that

$$
\inf \{h(t, z)+d(x, z): z \in Z\}=\inf _{j}\left[h\left(t, \psi_{j}(t)\right)+d\left(x, \psi_{j}(t)\right)\right] \quad \forall x \in Z, t \in T .
$$

Proof. We consider the sets

$$
S_{k, m}=\left\{(t, z) \in T \times B_{m}: h(t, z) \in U_{k}\right\},
$$

where $\left\{U_{k}\right\}$ is the sequence of all rational semiclosed intervals $(a, b]$ in $[-1,1]$ and $\left\{B_{m}\right\}$ is the sequence of all closed balls with positive rational radii centered at the points of a fixed countable dense set $\left\{z_{l}\right\}$ in $Z$. The sets $S_{k, m}$ are Borel. We take 
into account only nonempty sets $S_{k, m}$. Note that if $(t, z) \in S_{k, m}$, then $z$ must belong to $Z_{t}$, since $U_{k} \subset[-1,1]$ and $h(t, \cdot)=2$ outside $Z_{t}$. For each $t \in T$, the section

$$
S_{k, m}^{t}=\left\{x:(t, x) \in S_{k, m}\right\}
$$

is the difference of two compact sets by the lower semicontinuity of $h$ in the second argument and the inclusion $S_{k, m}^{t} \subset Z_{t}$. Hence this section is $\sigma$-compact. Therefore, by Theorem A stated above, the projection of $S_{k, m}$ onto $T$, denoted by $T_{k, m}$, is a Borel set and there is a Borel mapping $\psi_{k, m}: T_{k, m} \rightarrow Z$ such that $\psi_{k, m}(t) \in S_{k, m}^{t}$ for each $t \in T_{k, m}$. Outside $T_{k, m}$ we set $\psi_{k, m}(t)=z_{1}$. Let us add to this sequence the countable family of constant mappings with values in $\left\{z_{l}\right\}$. Finally, we renumber the obtained collection by using a single index $j$.

We now verify (5.2). Since both sides of (5.2) are Lipschitz in $x$, it suffices to show that they coincide for all $x \in\left\{z_{l}\right\}$. Fix $t \in T, x=z_{l}$ and $\varepsilon>0$. Take $z \in Z$ for which $h(t, z)+d(x, z)-\varepsilon / 2$ is less than the left-hand side of (15.2). If $x \notin Z_{t}$, then either the left-hand side equals 2 and the minimum is attained at $z=z_{l}$, so the corresponding constant function works, or $z \in Z_{t}$, because $h(t, z)=2$ outside $Z_{t}$. If $x \in Z_{t}$, then we also have $z \in Z_{t}$. We show that there are numbers $k$ and $m$ such that the left-hand side of (15.2) is larger than

$$
h\left(t, \psi_{k, m}(t)\right)+d\left(x, \psi_{k, m}(t)\right)-\varepsilon .
$$

To this end, we find $k$ and $m$ for which $h(t, z) \in U_{k}$ and $z \in B_{m}$, moreover, we pick $k$ and $m$ such that the length of $U_{k}$ and the diameter of $B_{m}$ are less than $\varepsilon / 8$. Then for the corresponding $\psi_{k, m}(t)$ we have $\psi_{k, m}(t) \in B_{m}, h\left(t, \psi_{k, m}(t)\right) \in U_{k}$, so that

$h\left(t, \psi_{k, m}(t)\right)+d\left(x, \psi_{k, m}(t)\right)<h(t, z)+d(x, z)+\varepsilon / 4<\inf \{h(t, z)+d(x, z): z \in Z\}+\varepsilon$, which completes the proof.

Lemma 5.6. Under the hypotheses of the previous lemma, there is a sequence of Borel functions $h_{n}: T \times Z \rightarrow[0,2]$ such that $h_{n} \leq h_{n+1}, h(t, z)=\lim _{n \rightarrow \infty} h_{n}(t, z)$, and the functions $z \mapsto h_{n}(t, z)$ are bounded Lipschitz for each $t$.

Proof. There is a classical construction for approximations:

$$
h_{n}(t, z)=\inf \{h(t, y)+n d(z, y), y \in Z\} .
$$

The function $h_{n}$ is Lipschitz in $z$ and $h_{n} \leq h$. Its Borel measurability in $t$ follows by the previous lemma applied to the metric $n d$, so $h_{n}$ is jointly Borel measurable.

Remark 5.7. The assumption that $T$ is a Souslin space has been used in the previous two lemmas to cover the case of lower semicontinuous functions. If the functions $h_{t}$ are continuous for each $t$ and $h$ is measurable on $T \times Z$ (not necessarily bounded), then both lemmas are valid for arbitrary measurable spaces $(T, \mathcal{T})$, since the approximations

$$
h_{n}(t, z)=\inf _{k}\left[h\left(t, y_{k}\right)+n d\left(z, y_{k}\right)\right],
$$

where $\left\{y_{k}\right\}$ is a fixed sequence dense in $Z$, coincide with the functions defined above by the infimum over the whole space and are Lipschitz. Replacing them by $\min \left(h_{n}, n\right)$ we obtain bounded Lipschitz functions increasing to $h$ and measurable on $T \times Z$.

Lemma 5.8. Suppose that lower semicontinuous cost functions $h_{n} \geq 0$ increase pointwise to a function $h$ for which $K_{h}(\mu, \nu)<\infty$. Let $\pi_{n} \in \Pi(\mu, \nu)$ be optimal 
measures for $h_{n}$ converging weakly to a Radon measure $\pi$. Then $\pi$ is an optimal measure for the triple $h, \mu, \nu$. In addition, $K_{h}(\mu, \nu)=\lim _{n \rightarrow \infty} K_{h_{n}}(\mu, \nu)=\lim _{n \rightarrow \infty} I_{h_{n}}\left(\pi_{n}\right)$.

Proof. For continuous cost functions this assertion is simple. For the reader's convenience, we include the proof. Clearly, $\pi \in \Pi(\mu, \nu)$. The sequence $\left\{\pi_{n}\right\}$ is uniformly tight, so, given $\varepsilon>0$, there is a compact set $K$ with $\pi(K)>1-\varepsilon, \pi_{n}(K)>1-\varepsilon$ for all $n$. Enlarging $K$ we can assume that the integral of $h$ over the complement of $K$ with respect to $\pi$ is less than $\varepsilon$. On $K$ convergence is uniform by Dini's theorem. Then

$$
\left|I_{h}(\pi)-K_{h_{n}}(\mu, \nu)\right| \leq 2 \varepsilon
$$

for large $n$. Hence the numbers $K_{h_{n}}(\mu, \nu)$ increase to $I_{h}(\pi)$. Since

$$
K_{h_{n}}(\mu, \nu) \leq K_{h}(\mu, \nu) \leq I_{h}(\pi),
$$

we have $I_{h}(\pi)=K_{h}(\mu, \nu)$. This reasoning also applies to the case where only the function $h$ is continuous, but all $h_{n}$ are lower semicontinuous (to apply Dini's theorem, we need the upper semicontinuity of the functions $h-h_{n}$ ).

Our next step is to observe that for lower semicontinuous $h$ the quantity $K_{h}(\mu, \nu)$ coincides with the supremum of $K_{w}(\mu, \nu)$ over bounded continuous cost functions $w \geq 0$ such that $w(x, y) \leq h(x, y)$ for all $x$ and $y$. This follows by the Kantorovich duality: for each $\varepsilon>0$ there are functions $\varphi \in C_{b}(X)$ and $\psi \in C_{b}(Y)$ such that

$$
\varphi(x)+\psi(y) \leq h(x, y)
$$

for all $x$ and $y$ and

$$
\int \varphi d \mu+\int \psi d \nu \geq K_{h}(\mu, \nu)-\varepsilon
$$

We now take $w(x, y)=\max (\varphi(x)+\psi(y), 0)$. Since $w(x, y) \geq \varphi(x)+\psi(y)$, the integral of $h$ against any measure in $\Pi(\mu, \nu)$ is at least $K_{h}(\mu, \nu)-\varepsilon$. Hence we have $K_{w}(\mu, \nu) \geq K_{h}(\mu, \nu)-\varepsilon$.

It follows that there is a pointwise increasing sequence of nonnegative functions $w_{n} \in C_{b}(X \times Y)$ such that $w_{n}(x, y) \leq h(x, y)$ and $K_{w_{n}}(\mu, \nu) \rightarrow K_{h}(\mu, \nu)$. Such functions can be found converging to $h$, since there is a sequence of bounded continuous functions $u_{n} \geq 0$ increasing to $h$, so we can take $\max \left(w_{n}, u_{n}\right)$ and observe that $K_{w_{n}}(\mu, \nu) \leq K_{\max \left(w_{n}, u_{n}\right)}(\mu, \nu) \leq K_{h}(\mu, \nu)$.

Let us show that there is no gap between $K_{h}(\mu, \nu)$ and the limit of $K_{h_{n}}(\mu, \nu)$ in the general case. Let $\varepsilon>0$. Take a function $w \in C_{b}(X \times Y)$ with $0 \leq w \leq h$ and $K_{w}(\mu, \nu) \geq K_{h}(\mu, \nu)-\varepsilon$.

The sequence of bounded lower semicontinuous functions $v_{n}=\min \left(w, h_{n}\right)$ increases pointwise to the bounded continuous function $w$. Hence by the previous step

$$
K_{v_{n}}(\mu, \nu) \rightarrow K_{w}(\mu, \nu) \geq K_{h}(\mu, \nu)-\varepsilon .
$$

Since $K_{h_{n}}(\mu, \nu) \geq K_{v_{n}}(\mu, \nu)$, we conclude that $K_{h_{n}}(\mu, \nu) \rightarrow K_{h}(\mu, \nu)$.

It remains to show that $K_{h}(\mu, \nu)$ coincides with $I_{h}(\pi)$. Otherwise for some $\delta>0$ we have $I_{h}(\pi)>K_{h}(\mu, \nu)+\delta$. Using the functions $w_{n}$ constructed above, we obtain a number $N$ such that

$$
\int w_{N} d \pi>K_{h}(\mu, \nu)+\delta / 2
$$

Hence

$$
\int w_{N} d \pi_{n}>K_{h}(\mu, \nu)+\delta / 2
$$


for all $n$ large enough. Since $w_{N}$ is bounded and $\left\{\pi_{n}\right\}$ is uniformly tight, there is a compact set $K$ such that

$$
\int_{K} w_{N} d \pi_{n}>K_{h}(\mu, \nu)+\delta / 4
$$

for all $n$ large enough. The functions $\min \left(h_{n}, w_{N}\right)$ are lower semicontinuous and increase to the continuous function $w_{N}$. Hence convergence is uniform on $K$. Therefore,

$$
\int_{K} \min \left(h_{n}, w_{N}\right) d \pi_{n}>K_{h}(\mu, \nu)+\delta / 8
$$

for all $n$ large enough. This yields the bound

$$
K_{h_{n}}(\mu, \nu)=\int_{X \times Y} h_{n} d \pi_{n} \geq \int_{K} h_{n} d \pi_{n} \geq \int_{K} \min \left(h_{n}, w_{N}\right) d \pi_{n}>K_{h}(\mu, \nu)+\delta / 8,
$$

which is a contradiction.

Lemma 5.9. Suppose that in the situation of Theorem 4.2 the measurability of $t \mapsto K_{h_{t}}\left(\mu_{t}, \nu_{t}\right)$ is given in advance. Then the assertion about the existence of a Borel version of $\sigma_{t}$ is true.

Proof. Now by assumption the function

$$
K(t)=K_{h_{t}}\left(\mu_{t}, \nu_{t}\right)
$$

is measurable on $T$. Let

$$
M_{t}:=\left\{\sigma \in \Pi\left(\mu_{t}, \nu_{t}\right): \int h(t, x, y) \sigma(d x d y)=K(t)\right\} .
$$

Each set $M_{t}$ is compact in $\Pi\left(\mu_{t}, \nu_{t}\right) \subset \mathcal{P}(X \times Y)$, because if measures $\sigma_{n} \in M_{t}$ converge weakly to a measure $\sigma$, then $\sigma \in \Pi\left(\mu_{t}, \nu_{t}\right)$ and the integral of $h_{t}$ against $\sigma$ cannot be larger than $K(t)$ by the lower semicontinuity of $h_{t}$, but obviously it cannot be smaller than $K(t)$ by the definition of $K(t)$.

By the Borel measurability of the function $t \mapsto K(t)$ and the Borel measurability of the function

$$
(t, \sigma) \mapsto \int_{X \times Y} h(t, x, y) \sigma(d x d y)
$$

on $T \times \mathcal{P}(X \times Y)$, which follows by the joint measurability of $h$ (see [12, Theorem 5.8.4]), the set

$$
B=\left\{(t, \sigma): \sigma \in \mathcal{P}(X \times Y), \sigma \in \Pi\left(\mu_{t}, \nu_{t}\right), \quad \int h(t, x, y) \sigma(d x d y)=K(t)\right\}
$$

is Borel in $T \times \mathcal{P}(X \times Y)$ and $M_{t}$ is its section at $t$. Hence again Theorem A applies.

Lemma 5.10. Let $(T, \mathcal{T})$ be a measurable space, let $E$ be a completely regular Souslin space, and let $u_{n}: T \rightarrow E$ be a sequence of $\mathcal{T}$-measurable mappings such that the sequence $\left\{u_{n}(t)\right\}$ has compact closure for every fixed $t \in T$. Then there is a sequence of $\mathcal{T}$-measurable functions $t \mapsto \eta_{k}(t)$ with values in $\mathbb{N}$ such that, for every $t$, the numbers $\eta_{k}(t)$ increase to infinity and the sequence $\left\{u_{\eta_{k}(t)}(t)\right\}$ converges to some point $u(t)$ such that the mapping $t \mapsto u(t)$ is $\mathcal{T}$-measurable. 
Proof. There is a continuous injection of $E$ into $[0,1]^{\infty}$, so we can consider $E$ as a set in $[0,1]^{\infty}$ with a stronger Souslin topology. Points of $[0,1]^{\infty}$ will be written as $x=\left(x^{1}, x^{2}, \ldots\right)$. It suffices to pick increasing numbers $\eta_{k}(t)$ measurably in $t$ in such a way that, for each $j$ and $t$, the sequence of numbers $u_{\eta_{k}(t)}^{j}(t)$ will converge. Indeed, this convergence implies that the sequence $\left\{u_{k(t)}(t)\right\}$ cannot have different limit points, but by the compactness of the closure this sequence must have limit points, so it follows that the whole sequence converges.

We construct $\eta_{k}(t)$ inductively. By the measurability of $u_{n}$ the functions

$$
L_{j}(t)=\limsup _{n \rightarrow \infty} u_{n}^{j}(t)
$$

are $\mathcal{T}$-measurable. Let $\eta_{1}^{1}(t)$ be the minimal number $n$ such that

$$
\left|u_{n}^{1}(t)-L_{1}(t)\right|<1 \text {. }
$$

This number measurably depends on $t$, because

$$
\begin{aligned}
& \left\{t \in T: \eta_{1}^{1}(t)=m\right\} \\
& =\left\{t:\left|u_{n}^{1}(t)-L_{1}(t)\right| \geq 1, n=1, \ldots, m-1,\left|u_{m}^{1}(t)-L_{1}(t)\right|<1\right\} .
\end{aligned}
$$

Assuming that $\eta_{k}^{1}(t)$ is already defined and $\mathcal{T}$-measurable, we take for $\eta_{k+1}^{1}(t)$ the minimal number $n$ such that $n>\eta_{k}^{1}(t)$ and

$$
\left|u_{n}^{1}(t)-L_{1}(t)\right|<\frac{1}{k+1} .
$$

As above, the function $\eta_{k+1}^{1}$ is $\mathcal{T}$-measurable. It follows that the first coordinates of $u_{\eta_{k}^{1}(t)}$ converge to $L_{1}(t)$.

The second step is to pick a subsequence in $\left\{\eta_{k}^{1}(t)\right\}$ for which the second coordinates will converge to $L_{2}(t)$. To this end, we take for $\eta_{1}^{2}(t)$ the minimal number $n>\eta_{1}^{1}(t)$ among the numbers $\eta_{k}^{1}(t)$ such that

$$
\left|u_{n}^{2}(t)-L_{2}(t)\right|<1 \text {. }
$$

We have

$$
\begin{aligned}
& \left\{t \in T: \eta_{1}^{2}(t)=m\right\} \\
& =\left\{t:\left|u_{\eta_{1}^{n}(t)}^{2}(t)-L_{2}(t)\right| \geq 1, n=1, \ldots, m-1,\left|u_{\eta_{m}^{1}(t)}^{2}(t)-L_{2}(t)\right|<1\right\},
\end{aligned}
$$

which shows that $\eta_{1}^{2}$ is $\mathcal{T}$-measurable. We proceed inductively and find $\mathcal{T}$-measurable functions $\eta_{k}^{2}$ such that $\eta_{k}^{2}(t)$ is the minimal number in $\left\{\eta_{k}^{1}(t)\right\}$ for which the difference between $L_{2}(t)$ and the second coordinate of $u_{\eta_{n}^{1}(t)}(t)$ becomes less than $1 / k$.

We continue this process inductively and obtain embedded subsequence $\left\{\eta_{k}^{m}(t)\right\}$ such that the functions $\eta_{k}^{m}$ are $\mathcal{T}$-measurable and the $m$ th coordinates of $u_{\eta_{k}^{m}(t)}(t)$ converge to $L_{m}(t)$. For the diagonal sequence $\eta_{k}^{k}(t)$ we have convergence of all coordinates, which proves convergence of $u_{\eta_{k}^{k}(t)}(t)$.

Corollary 5.11. Let $(T, \mathcal{T})$ be a measurable space, let $X$ be a completely regular Souslin space, and let $t \mapsto \mu_{t, n}, T \rightarrow \mathcal{M}(X)$ be a sequence of $\mathcal{T}$-measurable mappings such that the sequence of measures $\left\{\mu_{t, n}\right\}$ has weakly compact closure (for example, is uniformly tight) for every fixed $t \in T$. Then there is a sequence of $\mathcal{T}$-measurable functions $t \mapsto \eta_{k}(t)$ with values in $\mathbb{N}$ such that, for every $t$, the numbers $\eta_{k}(t)$ increase 
to infinity and the sequence of measures $\mu_{t, \eta_{k}(t)}$ converges to some measure $\mu_{t}$ such that $t \mapsto \mu_{t}$ is $\mathcal{T}$-measurable.

Proof. The previous lemma applies, since the space of measures on $X$ with the weak topology is also Souslin.

Proof of Theorem 4.1. By Corollary 5.11 for completing the proof of Theorem 4.1 it suffices to find approximate $\mathcal{T}$-measurable solutions $\sigma_{t, n}$ with $I_{h}\left(\sigma_{t, n}\right) \rightarrow K(t)$ for each $t$. To this end, we find $\mathcal{T}$-measurable solutions $\pi_{t, n}$ for bounded Lipschitz cost functions $h_{n}$ increasing to $h$ and constructed according to Remark 5.7. Therefore, the general case reduces to the case in which every function $h_{t}$ is bounded by 1 and Lipschitz with constant 1. Moreover, by Lemma 5.2 and Lemma 5.4 it suffices to consider the case in which the measures $\mu_{t}$ and $\nu_{t}$ have compact supports, so that for each $t$ there is a compact set $S_{t}$ on which all measures from $\Pi\left(\mu_{t}, \nu_{t}\right)$ are concentrated and $S_{t}$ depends on $t$ measurably.

Let us consider the space $\mathcal{K}(X \times Y)$ of nonempty compact subsets of $X \times Y$ with the Hausdorff distance $d_{H}$ introduced in Section 2. This space is separable, hence there is a sequence of compacts sets $Q_{j}$ dense in the union of $S_{t}$. Let fix $n$ and consider the sets

$$
T_{j}=\left\{t \in T: \operatorname{dist}_{H}\left(S_{t}, Q_{j}\right) \leq 1 / n\right\} .
$$

Note that $T_{j} \in \mathcal{T}$ (this follows from the proof of Lemma 5.2). The set of 1-Lipschitz functions on $Q_{j}$ with values in $[0,1]$ is compact in the sup-norm, hence there is a sequence $h_{j, m}$ dense in it. Each function $h_{j, m}$ has an extension (denoted by the same symbol) to all of $X \times Y$ with values in $[0,1]$ and 1-Lipschitz.

We further define the sets

$$
T_{j, m}=\left\{t \in D_{j}: \sup _{(x, y) \in Q_{j}}\left|h_{t}(x, y)-h_{j, m}(x, y)\right| \leq 1 / n\right\} .
$$

The supremum can be taken over a countable set dense in $Q_{j}$, hence $T_{j, m} \in \mathcal{T}$. Using these sets we obtain a partition of $T$ into nonempty disjoint sets $D_{k} \in \mathcal{T}$ with the following property: for each $D_{k}$ there are numbers $j$ and $m$ such that $\operatorname{dist}_{H}\left(S_{t}, Q_{j}\right) \leq 1 / n$ and $\sup _{(x, y) \in Q_{j}}\left|h_{t}(x, y)-h_{j, m}(x, y)\right| \leq 1 / n$ for all $t \in D_{k}$. In every set $D_{k}$ take a point $t_{k}$. The cost function $h_{t_{k}}$ differs from any other cost function $h_{t}$ with $t \in D_{k}$ by at most $3 / n$ on the set $S_{t}$. Indeed, if $(x, y) \in S_{t}$, then we can find $(u, v) \in Q_{j}$ with $d((x, y),(u, v)) \leq 1 / n$. Since on $Q_{j}$ the functions $h_{t}$ and $h_{t_{k}}$ differ by at most $1 / n$, we have

$$
\begin{aligned}
& \left|h_{t}(x, y)-h_{t_{k}}(x, y)\right| \\
& \quad \leq\left|h_{t}(x, y)-h_{t}(u, v)\right|+\left|h_{t}(u, v)-h_{t_{k}}(u, v)\right|+\left|h_{t_{k}}(u, v)-h_{t_{k}}(x, y)\right| \leq 3 n^{-1} .
\end{aligned}
$$

Finally, on each $D_{k}$ we solve the Kantorovich problem with the cost function $h_{t_{k}}$ independent of $t$ and the original marginals. Hence there is a solution $\pi_{t}^{k} \in \Pi\left(\mu_{t}, \nu_{t}\right)$ that is $\mathcal{T}$-measurable. Clearly, $\left|K_{h_{t}}\left(\mu_{t}, \nu_{t}\right)-K_{h_{k}}\left(\mu_{t}, \nu_{t}\right)\right| \leq 3 / n$ for all $t \in D_{k}$. Therefore, on all of $T$ we obtain the desired approximation.

Proof of Theorem 4.2. By Lemma 5.9 it suffices to prove the Borel measurability of the transportation cost $K_{t}=K_{h_{t}}\left(\mu_{t}, \nu_{t}\right)$. Using Lemma 5.8 and the truncations $\min \left(h_{t}, N\right)$ we can pass to uniformly bounded cost functions. So we can assume that $h_{t}<1$. Lemma 5.4 reduces the assertion to the case of measures $\mu_{t}^{n}$ and $\nu_{t}^{n}$ with compact supports $Z_{1}^{n}(t)$ and $Z_{2}^{n}(t)$. The value of the cost does not change if we redefine $h_{t}$ outside $Z_{1}^{n}(t) \times Z_{2}^{n}(t)$ by the value 2 . Since the set $\bigcup_{t}\left(\{t\} \times Z_{1}^{n}(t) \times Z_{2}^{n}(t)\right)$ 
belongs to $\mathcal{T} \otimes \mathcal{B}(X) \otimes \mathcal{B}(Y)$ by Lemma 5.2, this new cost function is Borel. It is readily seen that it is lower semicontinuous.

Now we are in the situation of Lemma 5.6. Therefore, Lemma 5.8 further reduces everything to continuous cost functions. This case is covered by the first (and easy) part of Theorem 4.1.

Remark 5.12. (i) As already noted in the introduction, Zhang [43] proved that if cost functions $h_{t}$ are continuous, then the space $M$ of nonnegative continuous cost functions can be regarded as a parametric space and equipped with its natural Borel $\sigma$-algebra (generated by the metric introduced above) and the set-valued mapping $(h, \mu, \nu) \mapsto \operatorname{Opt}(h, \mu, \nu)$ has a Borel measurable selection.

However, it is not clear how this can be applied to the assertion announced in [43] that a measurable selection exists for any parametric measurable space $(T, \mathcal{T})$. The point is that the mapping $t \mapsto h_{t}$ with values in $M$ generated by a function $h$ Borel measurable in $t$ can fail to be measurable when $M$ is equipped with the Borel $\sigma$-algebra. For example, this happens if $T=C_{b}(B \times B)$ with its sup-norm, where $B$ is the unit ball in $l^{2}, \mathcal{T}$ is generated by evaluation functionals $t \mapsto t(x, y)$, $X=Y=B$, and $h(t, x, y)=t(x, y)$. Here $h$ is bounded and continuous in $(x, y)$ and $\mathcal{T}$-measurable, but $t \mapsto h_{t}$ is not measurable with values in $C_{b}(B \times B)$ equipped with the Borel $\sigma$-algebra.

To see this, let us observe that the Borel $\sigma$-algebra of the space $C_{b}(\mathbb{N})$ is not countably generated, because its cardinality is greater than that of the continuum. Indeed, this space contains a closed discrete set of cardinality of the continuum; all subsets of this set are also closed. It follows that $\mathcal{B}\left(C_{b}(\mathbb{N})\right)$ is not generated by the evaluation functionals $f \mapsto f(n)$. The same is true for any metric space containing a discrete countable subset, hence for any noncompact metric space. Similarly, the Borel $\sigma$-algebra of the metric space $M=C(X \times Y)$ mentioned in the introduction is not generated by evaluation functionals if the balls in $X \times Y$ are not compact.

However, for any compact metric space $K$ the Borel $\sigma$-algebra of the space $C_{b}(K)$ is generated by the evaluation functionals $f \mapsto f(k)$, because this space is separable and these functionals separate its points. Hence the proof in [43] for general $(T, \mathcal{T})$ is correct if $X$ is a locally compact Polish space. We have not succeeded to fix the general case in a simple way and needed several steps. Recall also that for lower semicontinuous cost functions we still assume that $T$ is Souslin.

(ii) The question also arises whether Theorem 4.2 extends to Souslin spaces $X$ and $Y$. A major problem is to extend Lemma 5.4 to Souslin spaces $X$. Suppose that for a lower semicontinuous $h_{t}$ and Souslin spaces $X$ and $Y$ we know that there are measurable set-valued mappings $t \mapsto Z_{n}(t)$ as in Lemma 5.4. We take a bounded continuous metric $d$ on $X \times Y$ and observe that the functions $h_{k}(t, x, y)=\inf \left\{h(t, u, v)+k d((x, y),(u, v)):(u, v) \in Z_{n}(t)\right\}$ increase on $Z_{n}(t)$ to $h(t, x, y)$, because on $Z_{n}(t)$ the topology of $X \times Y$ is metrizable by $d$ by compactness. Moreover, the assumed measurability of $Z_{n}(t)$ ensures (for each fixed $n$ ) the existence of a sequence of measurable mappings $\xi_{j}: T \rightarrow X \times Y$ such that $\xi_{j}(t) \in Z_{n}(t)$ and $Z_{n}(t)$ is the closure of $\left\{\xi_{j}(t)\right\}$. So the infimum defining $h_{k}(t, x, y)$ can be evaluated over $\left\{\xi_{j}(t)\right\}$, which shows the measurability of $h_{k}(t, x, y)$.

Of course, if we agree to leave the safe area of Borel measurability, for Souslin spaces it is possible to impose the following stronger condition on $\mu_{t}$ and $\nu_{t}$ : let these mappings be measurable when $X$ and $Y$ are equipped with the $\sigma$-algebras $\sigma(\mathcal{S}(X))$ 
and $\sigma(\mathcal{S}(X))$. Then $K(t)$ is $\sigma(\mathcal{S}(T))$-measurable and $\sigma_{t}$ can be made $\sigma(\mathcal{S}(T))$ measurable. Indeed, there are continuous surjections $g_{1}: \mathbb{R}^{\infty} \rightarrow X, g_{2}: \mathbb{R}^{\infty} \rightarrow Y$. Hence we obtain two families $\mu_{t}^{1}=\mu_{t} \circ g_{1}^{-1}, \nu_{t}^{2}=\nu_{t} \circ g_{2}^{-1}$ of measures on $\mathbb{R}^{\infty}$ that are $\sigma(\mathcal{S}(T)$ )-measurable. The obtained results apply to these measures and the cost function $h^{0}(t, u, v)=h\left(t, g_{1}(u), g_{2}(u)\right)$, which satisfies our hypotheses. The corresponding transportation cost and optimal measures will be $\sigma(\mathcal{S}(T))$-measurable. Then we take the images of optimal measures under the mapping $\left(g_{1}, g_{2}\right)$.

Closing this section we mention that similar results can be obtained for the Kantorovich problem with density constraints studied by Korman and McCann [31] (see also [20]). The density constraint is an additional requirement on admissible optimal measures: in place of the set $\Pi(\mu, \nu)$ we consider its subset $\Pi^{\theta}(\mu, \nu)$ consisting of measures having densities with respect to a given measure $\lambda$ on $X \times Y$ bounded by a given nonnegative Borel function $\theta \in L^{1}(\lambda)$. If $\Pi^{\theta}(\mu, \nu)$ is not empty and the cost function is bounded and lower semicontinuous, then the set of minimizing measures is not empty. A straightforward modification of the reasoning above shows that also in this case there is a measurable choice of optimal measures depending on the parameter on which marginal measures and the cost function depend measurably. In a separate paper we shall consider a more general situation where the constraint $\theta$ and the reference measure $\lambda$ also depend on a parameter.

\section{The Skorohod PARAmetrization With A PARAMETER}

In this short section we consider another parametric problem in the same circle of ideas. It was shown by Skorohod [38] that for any weakly convergent sequence of Borel probability measures $\mu_{n}$ on a complete separable metric space $X$ there is a sequence of Borel mappings $\xi_{n}:[0,1] \rightarrow X$ with $\mu_{n}=\lambda \circ \xi_{n}^{-1}$, where $\lambda$ is Lebesgue measure, converging almost everywhere. This important result was generalized by Blackwell and Dubins [7] and Fernique [23], who proved that for every measure $\mu \in \mathcal{P}(X)$ there is a Borel mapping $\xi_{\mu}:[0,1] \rightarrow X$ such that $\mu$ is the image of Lebesgue measure $\lambda$ under $\xi_{\mu}$ and measures $\mu_{n}$ converge weakly to $\mu$ if and only if the mappings $\xi_{\mu_{n}}$ converge to $\xi_{\mu}$ almost everywhere. A topological proof of this result along with some generalizations was given in [13] (see also [4], [9], and [12] on this topic). The purpose of this section is to verify that this topological proof actually yields the following result.

Theorem 6.1. Let $X$ be a complete separable metric space. For every measure $\mu \in$ $\mathcal{P}(X)$ there is a Borel mapping $\xi_{\mu}:[0,1] \rightarrow X$ with $\mu=\lambda \circ \xi_{\mu}^{-1}$ such that the mapping $(\mu, t) \mapsto \xi_{\mu}(t)$ is Borel measurable on $\mathcal{P}(X) \times[0,1]$ and measures $\mu_{n}$ converge weakly to $\mu$ if and only if the mappings $\xi_{\mu_{n}}$ converge to $\xi_{\mu}$ almost everywhere.

Therefore, for any family of measures $\mu_{\omega} \in \mathcal{P}(X)$ measurably depending on a parameter $\omega$ from a measurable space $(\Omega, \mathcal{A})$, the mapping $(\omega, t)=\xi_{\mu_{\omega}}(t)$ with values in $X$ is $\mathcal{A} \otimes \mathcal{B}[0,1]$-measurable.

Proof. We verify that the proof suggested in [13] and also presented in [9, §8.5] and $[12, \S 2.6]$ gives the desired version. This proof is very simple. First we explicitly define the desired mapping for the space $X=[0,1]$ :

$$
\xi_{\mu}(t)=\sup \{x \in[0,1]: \mu([0, x)) \leq t\} .
$$

It is shown in [12, Theorem 2.6.4] that this is the desired parametrization. We only need to show that $\xi_{\mu}(t)$ is jointly Borel measurable on $\mathcal{P}([0,1]) \times[0,1]$. Note that 
$\xi_{\mu}(t)$ is increasing and right-continuous in $t$. It is known that if a function $\xi_{\mu}(t)$ is increasing and right-continuous in $t$ for every fixed $\mu$ and is Borel measurable in $\mu$ for each fixed $t$, then it is jointly Borel measurable. Indeed, it suffices to observe that it is the limit of the decreasing sequence of functions $\xi_{n}(\mu, t)$ defined as follows: for each $n$, we partition $[0,1]$ into $2^{n}$ intervals $I_{1}=\left[0,2^{-n}\right), I_{2}=\left[2^{-n}, 2^{2-n}\right), \ldots, I_{2^{n}}=$ $\left[1-2^{-n}, 1\right]$ and set $\xi_{n}(\mu, t)=\xi_{\mu}\left(r_{k}\right)$ if $t \in I_{k}$ and $r_{k}$ is the right end of $I_{k}$.

The next step is to observe that once this theorem is established for some space $X$, it remains valid for every Borel subspace $E \subset X$. Indeed, every measure $\mu \epsilon$ $\mathcal{P}(E)$ extends to a measure on $X$ by letting $\mu(X \backslash E)=0$. We take a jointly Borel measurable mapping $(\mu, t) \mapsto \xi_{\mu}(t)$ for $X$ and for measures concentrated on $E$ redefine it by $\eta_{\mu}(t)=\xi_{\mu}(t)$ if $\xi_{\mu}(t) \in E$ and $\eta_{\mu}(t)=x_{0}$ if $\xi_{\mu}(t) \notin E$, where $x_{0} \in E$ is a fixed element. Since $\xi_{\mu}(t) \in E$ for almost all $t$ for $\mu$ concentrated on $E$, we do not change the image of Lebesgue measure. The obtained mapping is obviously Borel measurable and gives the desired parametrization for $\mathcal{P}(E)$.

It follows from the previous step that the theorem is true for the Cantor set $C$. It is known that every compact metric space is the image of $C$ under some continuous mapping, in particular, there is a continuous surjection $h: C \rightarrow[0,1]^{\infty}$. Then the induced mapping $H: \mathcal{P}(C) \rightarrow \mathcal{P}\left([0,1]^{\infty}\right)$ defined by $H(\mu)=\mu \circ h^{-1}$ is also a continuous surjection. By the Milyutin theorem (see [12, §2.6] for details) there is a continuous affine mapping $G: \mathcal{P}\left([0,1]^{\infty}\right) \rightarrow \mathcal{P}(C)$ that is a right inverse for $H$, i.e., $H(G(\nu))=\nu$ for all $\nu \in \mathcal{P}\left([0,1]^{\infty}\right)$. Therefore, using a jointly Borel measurable parametrization $\xi_{\mu}(t)$ for $\mathcal{P}(C)$ we obtain a jointly Borel measurable parametrization $h\left(\xi_{G(\mu)}(t)\right)$ for $\mathcal{P}\left([0,1]^{\infty}\right)$. Hence the desired parametrization exists for every Borel subspace in $[0,1]^{\infty}$, but every Polish space is homeomorphic to a $G_{\delta^{-}}$set in $[0,1]^{\infty}$, see [21, Theorem 4.2.10, Theorem 4.3.24, Corollary 4.3.25], which completes the proof.

Remark 6.2. A drawback of convergence almost everywhere is that there is no topology in which convergent sequences are precisely the sequences converging almost everywhere. For this reason it may be more convenient to consider on the space of Borel mappings from $[0,1]$ to $X$ the semimetric of convergence in measure defined by

$$
d_{0}(\xi, \eta)=\int_{0}^{1} \min (d(\xi(t), \eta(t)), 1) d t
$$

where $d$ is a complete metric on $X$. The corresponding quotient space is also complete separable. It is clear that for the obtained parametrization convergence of mappings in this semimetric is equivalent to weak convergence of their laws. Actually, this parametrization gives a homeomorphism of the quotient space $L^{0}(\lambda, X)$ of $X$-valued mappings with convergence in measure and the space $\mathcal{P}(X)$.

Acknowledgements. This work has been supported by the Russian Science Foundation Grant 17-11-01058 at Lomonosov Moscow State University. The results presented in Section 6 were obtained within the project of the second author supported by the Foundation for the Advancement of Theoretical Physics and Mathematics "BASIS". We are very grateful to Sergey Kuksin and Armen Shirikyan for inspiring discussions and useful comments.

\section{REFERENCES}

[1] G.A. Alekseev, E.V. Yurova, On Gaussian conditional measures depending on a parameter, Theory Stoch. Processes 22 (2) (2017), 1-7. 
[2] L. Ambrosio, N. Gigli, A user's guide to optimal transport, Lecture Notes in Math. 2062 (2013), 1-155.

[3] J.-P. Aubin, H. Frankowska, Set-valued analysis. Birkhäuser Boston, Boston, 1990.

[4] T.O. Banakh, V.I. Bogachev, A.V. Kolesnikov, Topological spaces with the strong Skorokhod property, Georgian Math. J. 8 (2) (2001), 201-220.

[5] M. Beiglböck, C. Leonard, W. Schachermayer, On the duality theory for the MongeKantorovich transport problem, In: Optimal transportation, pp. 216-265. London Math. Soc. Lecture Note Ser., V. 413. Cambridge Univ. Press, Cambridge, 2014.

[6] M. Beiglböck, W. Schachermayer, Duality for Borel measurable cost functions, Trans. Amer. Math. Soc. 363 (8) (2011), 4203-4224.

[7] D. Blackwell, L.E. Dubins, An extension of Skorohod's almost sure representation theorem, Proc. Amer. Math. Soc. 89 (4) (1983), 691-692.

[8] D. Blackwell, C. Ryll-Nardzewski, Non-existence of everywhere proper conditional distributions, Ann. Math. Statist. 34 (1963), 223-225.

[9] V.I. Bogachev, Measure Theory, vols. 1, 2, Springer, Berlin, 2007.

[10] V.I. Bogachev, Differentiable Measures and the Malliavin Calculus, Amer. Math. Soc., Providence, Rhode Island, 2010.

[11] V.I. Bogachev, Surface measures in infinite-dimensional spaces, In: Measure theory in nonsmooth spaces, pp. 52-97, Partial Differ. Equ. Meas. Theory, De Gruyter Open, Warsaw, 2017.

[12] V.I. Bogachev, Weak Convergence of Measures, Amer. Math. Soc., Providence, Rhode Island, 2018.

[13] V.I. Bogachev, A.V. Kolesnikov, Open mappings of probability measures and the Skorohod representation theorem, Teor. Veroyatn. Primen. 46 (1) (2001), 3-27 (in Russian); English transl.: Theory Probab. Appl. 46 (1) (2001), 20-38.

[14] V.I. Bogachev, A.V. Kolesnikov, The Monge-Kantorovich problem: achievements, connections, and prospects, Uspekhi Matem. Nauk 67 (5) (2012), 3-110 (in Russian); English transl.: Russian Math. Surveys 67 (5) (2012), 785-890.

[15] V.I. Bogachev, I.I. Malofeev, Surface measures generated by differentiable measures, Potential Anal. 44 (4) (2016), 767-792.

[16] C. Castaing, P. Raynaud de Fitte, M. Valadier, Young Measures on Topological Spaces. With Applications in Control Theory and Probability Theory, Kluwer, Dordrecht, 2004.

[17] C. Castaing, M. Valadier, Convex Analysis and Measurable Multifunctions, Lecture Notes in Math. V. 580, Springer-Verlag, Berlin - New York, 1977.

[18] J. Dedecker, C. Prieur, P. Raynaud De Fitte, Parametrized Kantorovich-Rubinštein theorem and application to the coupling of random variables, In: Dependence in probability and statistics, pp. 105-121, Lect. Notes Stat., V. 187, Springer, New York, 2006.

[19] C. Dellacherie, Un cours sur les ensembles analytiques, In: Analytic sets, pp. 184-316. Academic Press, New York, 1980.

[20] A.N. Doledenok, On a Kantorovich problem with a density constraint. Mat. Zametki 104 (1) (2018), 45-55 (in Russian); English transl.: Math. Notes 104 (1) (2018), 39-47.

[21] P. Engelking, General Topology, Polish Sci. Publ., Warszawa, 1977.

[22] I.V. Evstigneev, Regular conditional expectations of random variables depending on parameters, Teor. Veroyatnost. i Primenen. 31 (3) (1986), 586-589 (in Russian); English transl.: Theory Probab. Appl. 31 (3) (1987), 515-518.

[23] X. Fernique, Un modèle presque sûr pour la convergence en loi, C. R. Acad. Sci. Paris, Sér. 1 306 (1988), 335-338.

[24] W. Gangbo, R.J. McCann, The geometry of optimal transportation, Acta Math. 177 (1996), $113-161$.

[25] P.-L. Hennequin, A. Tortrat, Théorie des Probabilités et Quelques Applications, Masson et Gie, Paris, 1965.

[26] J. Hille, D. Plachky, J. Roters, Versions of conditional expectations depending continuously on parameters, Math. Methods Statist. 8 (1) (1999), 99-108.

[27] J. Hoffmann-Jørgensen, Existence of conditional probabilities, Math. Scand. 28 (2) (1971), $257-264$.

[28] J. Hoffmann-Jørgensen, Probability with a View Toward Statistics, vols. I, II, Chapman \& Hall, New York, 1994. 
[29] A.S. Kechris, Classical Descriptive Set Theory, Springer, Berlin - New York, 1995.

[30] H.G. Kellerer, Duality theorems for marginal problems, Z. Wahrsch. verw. Geb. 67 (4) (1984), 399-432.

[31] J. Korman, R.J. McCann, Optimal transportation with capacity constraints, Trans. Amer. Math. Soc. 367 (3) (2015), 1501-1521.

[32] S. Kuksin, V. Nersesyan, A. Shirikyan, Exponential mixing for a class of dissipative PDEs with bounded degenerate noise, Arxiv 1802.03250v2.

[33] I.I. Malofeev, Measurable dependence of conditional measures on a parameter, Dokl. Akad. Nauk 470 (1) (2016), 13-17 (in Russian); English transl.: Dokl. Math. 94 (2) (2016), 493-497.

[34] J. Pfanzagl, Parametric Statistical Theory, Walter de Gruyter, Berlin, 1994.

[35] S.T. Rachev, L. Rüschendorf, Mass Transportation Problems, vols. I, II, Springer, New York, 1998.

[36] D. Ramachandran, A note on regular conditional probabilities in Doob's sense, Annals Probab. 9 (5) (1981), 907-908.

[37] M.M. Rao, Conditional Measures and Applications, 2nd ed. Chapman and Hall/CRC, Boca Raton, Florida, 2005.

[38] A.V. Skorohod, Limit theorems for stochastic processes, Teor. Veroyatn. Primen. 1 (1956), 261-290 (in Russian); English transl.: Theory Probab. Appl. 1 (1956), 261-290.

[39] T. Tjur, Conditional Probability Distributions, Lecture Notes, No. 2, Institute of Mathematical Statistics, University of Copenhagen, Copenhagen, 1974.

[40] B.E. Trumbo, Sufficient conditions for the weak convergence of conditional probability distributions in a metric space, Thesis (Ph.D.) The University of Chicago, 1965.

[41] C. Villani, Topics in Optimal Transportation, Amer. Math. Soc., Providence, Rhode Island, 2003.

[42] C. Villani, Optimal Transport, Old and New, Springer, New York, 2009.

[43] X. Zhang, Stochastic Monge-Kantorovich problem and its duality, Stochastics 85 (1) (2013), $71-84$. 